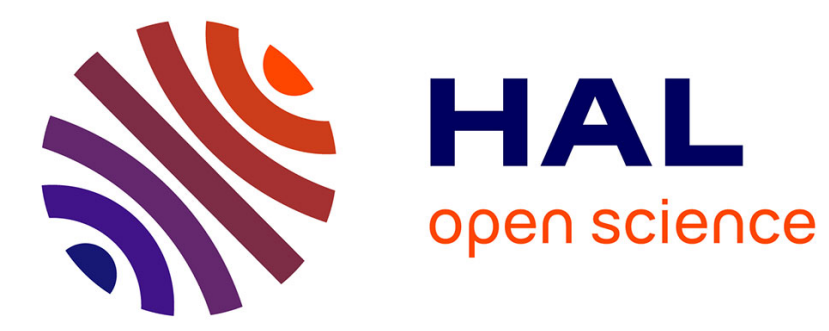

\title{
A POSTERIORI ANALYSIS OF ITERATIVE ALGORITHMS FOR A NONLINEAR PROBLEM
}

Christine Bernardi, Jad Dakroub, Gihane Mansour, Toni Sayah

\section{To cite this version:}

Christine Bernardi, Jad Dakroub, Gihane Mansour, Toni Sayah. A POSTERIORI ANALYSIS OF ITERATIVE ALGORITHMS FOR A NONLINEAR PROBLEM. 2013. hal-00918226v2

\section{HAL Id: hal-00918226 \\ https://hal.sorbonne-universite.fr/hal-00918226v2}

Preprint submitted on 18 Dec 2013

HAL is a multi-disciplinary open access archive for the deposit and dissemination of scientific research documents, whether they are published or not. The documents may come from teaching and research institutions in France or abroad, or from public or private research centers.
L'archive ouverte pluridisciplinaire HAL, est destinée au dépôt et à la diffusion de documents scientifiques de niveau recherche, publiés ou non, émanant des établissements d'enseignement et de recherche français ou étrangers, des laboratoires publics ou privés. 


\title{
A POSTERIORI ANALYSIS OF ITERATIVE ALGORITHMS FOR A NONLINEAR PROBLEM
}

\author{
CHRISTINE BERNARDI ${ }^{\dagger}$, JAD DAKROUB ${ }^{\dagger}$, GIHANE MANSOUR $\ddagger$, TONY SAYAH $\ddagger$.
}

\begin{abstract}
A posteriori error indicators have been studied in recent years owing to their remarkable capacity to enhance both speed and accuracy in computing. This work deals with a posteriori error estimation for the finite element discretization of a nonlinear problem. For a given nonlinear equation considering finite elements we solve the discrete problem using two iterative methods involving some kind of linearization. For each of them, there are actually two sources of error, namely discretization and linearization. Balancing these two errors can be very important, since it avoids performing an excessive number of iterations. Our results lead to the construction of computable upper indicators for the full error.

Several numerical tests are provided to evaluate the efficiency of our indicators.
\end{abstract}

RÉSumÉ. Les indicateurs d'erreur a posteriori ont été beaucoup considérés au cours des dernières années à cause de leurs capacités remarquables à améliorer la vitesse et la précision dans la résolution itérative des problèmes. Dans ce travail, notre but est d'appliquer cette méthode pour un problème non linéaire. Nous proposons alors deux algorithmes itératifs de résolution du problème et nous étudions la convergence de ces algorithmes vers la solution du problème discret. Une étape importante consiste à démontrer des estimations d'erreur a posteriori en distinguant les erreurs de linéarisation et de discrétisation. Nous présentons finalement quelques résultats d'expériences numériques.

Keywords : A posteriori error estimation, nonlinear problems, iterative methods.

\section{INTRODUCTION}

Many research works deal with the a posteriori analysis and the adaptive mesh-refinement for finite element discretization of elliptic problems, [11]. First, a posteriori analysis controls the overall discretization error of the problem and it provides error indicators which can be computed from the computed numerical solution and the given data of the problem. Once these error indicators are constructed, we prove their efficiency by bounding each indicator by the local error. This analysis was first introduced by I. Babuška [2], and developed by R. Verfürth [11]. In this work, we are interested in studying the following nonlinear problem.

Let $\Omega$ be an open polygon of $\mathbb{R}^{d}, d=2$, we consider

$$
\begin{aligned}
-\Delta u+\lambda|u|^{2 p} u=f & \text { in } \Omega, \\
u=0 & \text { on } \partial \Omega,
\end{aligned}
$$

where $\lambda$ and $p$ are two positive real numbers. The right-hand side $f$ belongs to $H^{-1}(\Omega)$, the dual of the Sobolev space $H_{0}^{1}(\Omega)$. Using $\mathcal{P}_{1}$ Lagrange finite elements, the discrete variational problem amounts to a system of nonlinear equations that are solved using an iterative method involving some kind of linearization. Thus, two sources of error appear, namely linearization and discretization. The main goal of this work is to balance these two sources of error. In fact, if the discretization error dominates then the nonlinear solver iterations is reduced. Therefore, our objective is to calculate a posteriori error estimates

December 12, 2013.

$\dagger$ Laboratoire Jacques-Louis Lions - C.N.R.S. et Université Paris VI, France.

$¥$ Département de Mathématiques - Université Saint-Joseph, Lebanon.

bernardi@ann.jussieu.fr, jad.dakroub@usj.edu.lb, mgihane@fs.usj.edu.lb, tsayah@fs.usj.edu.lb. 
distinguishing linearization and discretization errors in the context of an adaptive procedure. This type of analysis was introduced by A.-L. Chaillou and M. Suri [4, 5] for a general class of problems characterized by strongly monotone operators. It had been developed by L. El Alaoui, A. Ern and M. Vohralík [6] for a class of second-order monotone quasi-linear diffusion-type problems approximated by piecewise affine, continuous finite elements. In fact, the main difference between these two works is that in [6] they considered an iterative loop for the linearization procedure while in $[4,5]$ they replaced the nonlinear problem by a simplified linear model without considering any adaptive procedure.

Furthermore, in this work we present two different strategies for the linearization process, namely fixedpoint algorithm and Newton algorithm. Both strategies are iterative and the algorithm can be outlined as follows :

(1) On the given mesh, perform an iterative linearization until the stopping criterion is satisfied

(2) If the error is less than the desired precision, then stop, else refine the mesh adaptively and go to step (1).

Then, we compare these two algorithms. Actually, the Newton iteration version has faster convergence rates than the fixed-point iteration version but is more sensitive to the initial values.

An outline of the paper is as follows. In Section 2, we present the variational formulation of problem (1.1)(1.2). We introduce in Section 3 the discrete variational problem with the a priori estimate. The two different algorithms are studied in Section 4. The a posteriori analysis of the discretization of both fixed-

point algorithm and Newton algorithm is performed in Section 5. Section 6 is devoted to the numerical experiments.

\section{ANALYSIS OF THE MODEL}

We describe in this section the nonlinear problem (1.1)-(1.2) together with its variational formulation. First of all, we recall the main notion and results which we use later on. For a domain $\Omega$, denote by $L^{p}(\Omega)$ the space of measurable functions summable with power $p$. For $v \in L^{p}(\Omega)$, the norm is defined by

$$
\|v\|_{L^{p}(\Omega)}=\left(\int_{\Omega}|v(x)|^{p} d \mathbf{x}\right)^{1 / p} .
$$

Throughout this paper, we constantly use the classical Sobolev space

$$
W^{m, r}(\Omega)=\left\{v \in L^{r}(\Omega) ; \forall|k| \leq m, \partial^{k} v \in L^{r}(\Omega)\right\},
$$

where $k=\left(k_{1}, k_{2}\right)$ is a 2-tuple of positive integers such that $|k|=k_{1}+k_{2}$ and

$$
\partial^{k} v=\frac{\partial^{|k|} v}{\partial x_{1}^{k_{1}} \partial x_{2}^{k_{2}}} .
$$

$W^{m, r}(\Omega)$ is equipped with the semi-norm

$$
|v|_{m, r, \Omega}=\left(\sum_{|k|=m} \int_{\Omega}\left|\partial^{k} v\right|^{r} d \mathbf{x}\right)^{1 / r}
$$

and the norm

$$
\|v\|_{m, r, \Omega}=\left(\sum_{\ell=0}^{m}|v|_{\ell, r, \Omega}^{r} d \mathbf{x}\right)^{1 / r} .
$$

For $r=2$, we define the Hilbert space $H^{m}(\Omega)=W^{m, 2}(\Omega)$. In particular, we consider the following space

$$
H_{0}^{1}(\Omega)=\left\{v \in H^{1}(\Omega), v_{\left.\right|_{\partial \Omega}}=0\right\}
$$

and its dual space $H^{-1}(\Omega)$.

We recall the Sobolev imbeddings (see Adams [1], Chapter 3).

Lemma 2.1. For all $1 \leq j<\infty$ and $d=2$, there exists a positive constant $S_{j}$ such that

$$
\forall v \in H_{0}^{1}(\Omega), \quad\|v\|_{L^{j}(\Omega)} \leq S_{j}|v|_{1, \Omega} .
$$


Remark 2.2. For $d=3$, inequality (2.1) with standard definition of $H_{0}^{1}(\Omega)$ remains valid only for $j \leq 6$, whence the interest of working in dimension $d=2$.

The model problem (1.1)-(1.2) admits the equivalent variational formulation :

Find $u \in X$ such that

$$
\forall v \in X, \quad \int_{\Omega} \nabla u \nabla v d \mathbf{x}+\int_{\Omega} \lambda|u|^{2 p} u v d \mathbf{x}=\langle f, v\rangle
$$

with $X=H_{0}^{1}(\Omega)$.

Theorem 2.3. Problem (2.2) admits a unique solution $u \in X$.

Proof. We associate the following energy functional with problem $(2.2)$ :

$$
E(u)=\frac{1}{2} \int_{\Omega} \nabla u(x)^{2} d \mathbf{x}+\frac{\lambda}{2 p+2} \int_{\Omega}|u(x)|^{2 p} u(x)^{2} d \mathbf{x}-\langle f, u(x)\rangle,
$$

we then have the estimation

$$
E(u) \geq \frac{1}{2} \int_{\Omega}|\nabla u(x)|^{2} d x+\frac{\lambda}{2 p+2} \int_{\Omega}|u|^{2 p+2}-\|f\|_{-1, \Omega}\|u\|_{1, \Omega} .
$$

Then, the result follows from the energy minimization corollary (see [9], Chapter 3).

We now introduce the following technical Lemma :

Lemma 2.4. Let $a, b$ and $p$ be three real numbers. We have the following relation

$$
\left.|| a\right|^{p}-|b|^{p}|\leq p| a-b \mid\left(|a|^{p-1}+|b|^{p-1}\right) .
$$

Proof. The result follows from applying the mean value theorem to $f(x)=x^{p}$ with $x>0$.

Remark 2.5. In the sequel, we denote by $C, C^{\prime}, \ldots$ generic constants that can vary from line to line but are always independent of all discretization parameters.

\section{Finite element Discretization And the a priori estimate}

This section collects some useful notation concerning the discrete setting and the a priori estimate.

Let $\left(\mathcal{T}_{h}\right)_{h}$ be a regular family of triangulations of $\Omega$, in the sense that, for each $h$ :

- The union of all elements of $\mathcal{T}_{h}$ is equal to $\bar{\Omega}$.

- The intersection of two different elements of $\mathcal{T}_{h}$, if not empty, is a vertex or a whole edge of both triangles.

- The ratio of the diameter $h_{K}$ of any element $K$ of $\mathcal{T}_{h}$ to the diameter of its inscribed circle is smaller than a constant independent of $h$.

As usual, $h$ stands for the maximum of the diameters $h_{K}, K \in \mathcal{T}_{h}$.

Let $X_{h} \subset H_{0}^{1}(\Omega)$ be the $\mathcal{P}_{1}$ finite element space associated with $\mathcal{T}_{h}$, more precisely

$$
X_{h}=\left\{v_{h} \in H_{0}^{1}(\Omega), \forall K \in \mathcal{T}_{h}, v_{h_{\mid K}} \in \mathcal{P}_{1}(K)\right\},
$$

where $\mathcal{P}_{1}(K)$ stands for the space of restrictions to $K$ of affine functions on $\mathbb{R}^{2}$.

We then consider the following finite element discretization of Problem (2.2), obtained by the Galerkin method :

Find $u_{h} \in X_{h}$ such that

$$
\forall v_{h} \in X_{h}, \quad \int_{\Omega} \nabla u_{h} \nabla v_{h} d \mathbf{x}+\int_{\Omega} \lambda\left|u_{h}\right|^{2 p} u_{h} v_{h} d \mathbf{x}=\left\langle f, v_{h}\right\rangle .
$$

In order to prove the existence of a solution to problem (3.1), let us recall some results on the finite dimensional approximations of nonlinear problems due to Brezzi-Rappaz-Raviart theorem [3]. Next, we 
apply them to problem (3.1). Let $V$ and $W$ be two Banach spaces. We introduce a $C^{1}$ mapping $G: V \rightarrow W$ and a linear continuous mapping $S \in \mathcal{L}(W, V)$. We set :

$$
F(u)=u-S G(u) .
$$

We consider the finite dimensional approximation of a solution $u \in V$ of the equation $F(u)=0$ :

For $h>0$, we are given a finite dimensional subspace $V_{h}$ of the space $V$ and an operator $S_{h} \in \mathcal{L}\left(W ; V_{h}\right)$. We set for $u_{h} \in V_{h}$ :

$$
F_{h}\left(u_{h}\right)=u_{h}-S_{h} G\left(u_{h}\right) .
$$

The approximate problem consists on finding a solution $u_{h} \in V_{h}$ of the equation

$$
F_{h}\left(u_{h}\right)=0 .
$$

Then, we have the following theorem (see [3], Section 3 or [7], Chapter 4) :

Theorem 3.1. Assume that $G$ is a $C^{1}$ mapping from $V$ into $W$ with $D G$ Lipschitz-continuous, $S D G(u) \in$ $\mathcal{L}(V)$ is compact and $D F(u)$ is an isomorphism of $V$. In addition, we assume that for all $v \in V$

$$
\lim _{h \rightarrow 0}\left\|v-\Pi_{h} v\right\|_{V}=0,
$$

for some linear operator $\Pi_{h} \in \mathcal{L}\left(V ; V_{h}\right)$ and

$$
\lim _{h \rightarrow 0}\left\|S_{h}-S\right\|_{\mathcal{L}(W, V)}=0 .
$$

Then, there exist $h_{0}>0$ and a neighborhood $\mathcal{O}$ of the origin in $V$ such that, for any $h \leq h_{0}$, problem (3.3) admits a unique solution $u_{h}$ such that $u_{h}-u$ belongs to $\mathcal{O}$.

Furthermore, we have for some constant $M>0$, independent of $h$

$$
\left\|u_{h}-u\right\|_{V} \leq M\left(\left\|u-\Pi_{h} u\right\|_{V}+\left\|\left(S_{h}-S\right) G(u)\right\|_{V}\right) .
$$

In order to apply the Brezzi-Rappaz-Raviart theorem [3] to problem (3.1) we take $V=H_{0}^{1}(\Omega)$ and $W=H^{-1}(\Omega)$. We introduce the linear continuous mapping

$$
\begin{aligned}
S: W & \rightarrow V \\
f & \mapsto S f=w,
\end{aligned}
$$

where $w$ is the solution of the problem

$$
\left\{\begin{array}{cccc}
-\Delta w & = & f & \text { in } \Omega \\
w & = & 0 & \text { on } \partial \Omega .
\end{array}\right.
$$

It is readily checked that $S$ is the Riesz isomorphism, hence an isometry between $H^{-1}(\Omega)$ and $H_{0}^{1}(\Omega)$.

Lemma 3.2. The following stability property holds for any $f$ in $H^{-1}(\Omega)$

$$
|S f|_{1, \Omega} \leq\|f\|_{-1, \Omega} .
$$

We consider now the following $C^{1}$ mapping

$$
\begin{aligned}
G: V & \rightarrow W \\
w & \mapsto G(w)=f-\lambda|w|^{2 p} w
\end{aligned}
$$

and observe that problem $(2.2)$ can be written as follows

$$
u-S G(u)=0 .
$$

Lemma 3.3. There exists a real number $L>0$, and a neighborhood $\mathcal{V}$ of $u$ in $H_{0}^{1}(\Omega)$ such that the following Lipschitz property holds

$$
\forall w \in \mathcal{V}, \quad\|\mathcal{S}(D G(u)-D G(w))\|_{\mathcal{L}\left(H_{0}^{1}(\Omega)\right)} \leq L|u-w|_{1, \Omega} .
$$

Proof. We have

$$
\|\mathcal{S}(D G(u)-D G(w))\|_{\mathcal{L}\left(H_{0}^{1}(\Omega)\right)} \leq\|D G(u)-D G(w)\|_{\mathcal{L}\left(H^{-1}(\Omega)\right)}
$$


We observe that for any $z \in H_{0}^{1}(\Omega)$,

$$
D G(u) . z-D G(w) . z=2 \lambda p\left(|w|^{2 p-1} w-|u|^{2 p-1} u\right) z-\lambda\left(|w|^{2 p}-|u|^{2 p}\right) z .
$$

Using lemma 2.4 and combining (3.7) with (3.8) yields the desired property.

Since the operator $S D G(u) \in \mathcal{L}(V)$ is compact, it follows from the Fredholm's alternative that $D F(u)$ is an isomorphism of $V$ if the equation

$$
D F(u) \cdot w=0 \quad \text { with } w \in V
$$

has only the zero solution. Thus, since

$$
D G(u) \cdot w=-\left(2 \lambda p|u|^{2 p-1} u w+\lambda|u|^{2 p} w\right),
$$

we consider the following problem

$$
\left\{\begin{array}{cccc}
-\Delta w+2 \lambda p|u|^{2 p-1} u w+\lambda|u|^{2 p} w & = & g & \text { in } \Omega \\
w & = & 0 & \text { on } \partial \Omega,
\end{array}\right.
$$

with $g \in H^{-1}(\Omega), \lambda>0$.

we now introduce the following lemma that proves the uniqueness of the solution $w=0$.

Lemma 3.4. The equation $(I-S D G(u)) \cdot w=0$ admits a unique solution $w=0$.

Proof. The existence and uniqueness of a solution to (3.9) is easily established due to Lax-Milgram theorem. Then, the result follows from the fact that $w=0$ is obviously a solution of problem (3.9).

We denote by $S_{h}$ the operator which associate with any $f$ in $W$ the solution $u_{h}$ of the discrete linear problem,

$$
\begin{aligned}
S_{h}: W & \rightarrow V_{h} \\
f & \mapsto S_{h} f=w_{h},
\end{aligned}
$$

where $w_{h}$ satisfies,

$$
\forall v_{h} \in V_{h}, \quad \int_{\Omega} \nabla w_{h} \nabla v_{h} d \mathbf{x}=\int_{\Omega} f v_{h} d \mathbf{x} .
$$

We are now in a position to state the following corollary which relies on Theorem 3.1 and provides the $a$ priori error estimate:

Corollary 3.5. Let $u$ be the solution of (2.2). There exist a neighborhood of the origin in $V$ and a real number $h_{0}>0$ such that, for all $h \leq h_{0}$, problem (3.1) has a unique solution $u_{h}$ with $u_{h}-u$ in this neighborhood. Moreover, the following a priori error estimate holds

$$
\left\|u_{h}-u\right\|_{V} \leq M\left\{\left\|u-\Pi_{h} u\right\|_{V}+\left\|\left(S_{h}-S\right) G(u)\right\|_{V}\right\},
$$

where $M$ is a constant independent of $h$.

In addition, if $u \in H^{2}(\Omega)$, we have

$$
\left\|u_{h}-u\right\|_{1, \Omega} \leq C h\|u\|_{2, \Omega} .
$$

\section{ItERATIVE ALGORITHMS}

In this section, in order to solve our nonlinear discrete problem, we propose two different algorithms, namely the fixed-point algorithm and the Newton algorithm. One of the main advantages of the fixedpoint algorithm is that it converges to the unique fixed-point of the function for any starting point. However, if the initial guess is sufficiently close to the theoretical solution, Newton iteration version leads to much faster convergence rates than the fixed-point iteration version. We start by introducing the fixed-point algorithm. 
4.1. Fixed-point algorithm. Let $u_{h}^{0}$ be an initial guess. We introduce, for $i \geq 0$, the following algorithm :

Find $u_{h}^{i+1} \in V_{h}$ such that

$$
\forall v_{h} \in V_{h}, \quad\left(\nabla u_{h}^{i+1}, \nabla v_{h}\right)+\lambda\left(\left|u_{h}^{i}\right|^{2 p} u_{h}^{i+1}, v_{h}\right)=\left\langle f, v_{h}\right\rangle .
$$

It is readily checked that problem (4.1) has a unique solution that depends continuously on $f$ :

$$
\left|u_{h}^{i+1}\right|_{1, \Omega} \leq\|f\|_{-1, \Omega} .
$$

The proof of its convergence relies on the coercivity of the bilinear form.

Theorem 4.1. (The convergence Theorem). Let $u_{h}^{i+1}$ and $u_{h}$ be the solutions of the iterative problem and the discrete problem respectively. Then

$$
\left|u_{h}^{i+1}-u_{h}\right|_{1, \Omega} \leq C_{1}^{-1} C_{2}\left|u_{h}^{i}-u_{h}\right|_{1, \Omega},
$$

where,

$$
\begin{aligned}
& C_{1}=1-\lambda S_{2} S_{4} S_{8 p}^{2 p}\|f\|_{-1, \Omega}^{2 p}, \\
& C_{2}=4 \lambda p S_{2} S_{4} S_{8} S_{8(2 p-1)}^{2 p-1}\|f\|_{-1, \Omega}^{2 p} .
\end{aligned}
$$

Moreover, $\left(u_{h}^{i}\right)_{i}$ converges if $C_{1}>0$ and $C_{1}^{-1} C_{2}<1$.

Proof. First, taking $v_{h}=u_{h}$ in (3.1) we have the following estimation

$$
\left|u_{h}\right|_{1, \Omega} \leq\|f\|_{-1, \Omega} .
$$

We start now by subtracting (4.1) from (3.1). We obtain, for all $v_{h} \in X_{h}$,

$$
\left(\nabla\left(u_{h}^{i+1}-u_{h}\right), \nabla v_{h}\right)=\lambda\left(\left|u_{h}\right|^{2 p} u_{h}-\left|u_{h}^{i}\right|^{2 p} u_{h}^{i+1}, v_{h}\right) .
$$

The right-hand side can be written as follows :

$$
\lambda\left(\left|u_{h}\right|^{2 p} u_{h}-\left|u_{h}^{i}\right|^{2 p} u_{h}^{i+1}, v_{h}\right)=\lambda\left(\left(\left|u_{h}\right|^{2 p}-\left|u_{h}^{i}\right|^{2 p}\right) u_{h}, v_{h}\right)+\lambda\left(\left|u_{h}^{i}\right|^{2 p}\left(u_{h}-u_{h}^{i+1}\right), v_{h}\right) .
$$

From Lemma 2.4, we have

$$
\left.|| u_{h}\right|^{2 p}-\left|u_{h}^{i}\right|^{2 p}\left|\leq 2 p\left(\left|u_{h}^{i}\right|^{2 p-1}+\left|u_{h}\right|^{2 p-1}\right)\right| u_{h}^{i}-u_{h} \mid .
$$

We now estimate the two terms in the right-hand side of (4.5).

(i) The first term is bounded, using (2.1), (4.6) and the Cauchy-Schwarz inequality, as follows

$$
\begin{aligned}
\int_{\Omega}\left(\left|u_{h}\right|^{2 p}-\left|u_{h}^{i}\right|^{2 p}\right) u_{h} v_{h} d \mathbf{x} & \leq 2 p \int_{\Omega}\left(\left|u_{h}^{i}\right|^{2 p-1}+\left|u_{h}\right|^{2 p-1}\right)\left|u_{h}^{i}-u_{h}\left\|u_{h}\right\| v_{h}\right| d \mathbf{x} \\
& \leq 2 p\left\|\left|u_{h}^{i}\right|^{2 p-1}+\left|u_{h}\right|^{2 p-1}\right\|_{L^{8}(\Omega)}\left\|u_{h}^{i}-u_{h}\right\|_{L^{8}(\Omega)}\left\|u_{h}\right\|_{L^{4}(\Omega)}\left\|v_{h}\right\|_{L^{2}(\Omega)} \\
& \leq 2 p S_{2} S_{4} S_{8}\left\|\left|u_{h}^{i}\right|^{2 p-1}+\left|u_{h}\right|^{2 p-1}\right\|_{L^{8}(\Omega)}\left|u_{h}^{i}-u_{h}\right|_{1, \Omega}\left|u_{h}\right|_{1, \Omega}\left|v_{h}\right|_{1, \Omega} .
\end{aligned}
$$

Using (4.2) and (4.3), leads to

$$
\lambda\left(\left(\left|u_{h}\right|^{2 p}-\left|u_{h}^{i}\right|^{2 p}\right) u_{h}, v_{h}\right) \leq 4 \lambda p S_{2} S_{4} S_{8} S_{8(2 p-1)}^{2 p-1}\|f\|_{-1, \Omega}^{2 p}\left|u_{h}^{i}-u_{h}\right|_{1, \Omega}\left|v_{h}\right|_{1, \Omega} .
$$

(ii) We now estimate the second term in the right-hand side of (4.5). Cauchy-Schwarz inequality gives

$$
\begin{aligned}
\left(\left|u_{h}^{i}\right|^{2 p}\left(u_{h}-u_{h}^{i+1}\right), v_{h}\right) & \leq\left\|u_{h}^{i 2 p}\right\|_{L^{4}(\Omega)}\left\|u_{h}^{i+1}-u_{h}\right\|_{L^{4}(\Omega)}\left\|v_{h}\right\|_{L^{2}(\Omega)} \\
& \leq\left\|u_{h}^{i}\right\|_{L^{8 p}(\Omega)}^{2 p}\left\|u_{h}^{i+1}-u_{h}\right\|_{L^{4}(\Omega)}\left\|v_{h}\right\|_{L^{2}(\Omega)} .
\end{aligned}
$$

Applying (2.1) and (4.2), we obtain

$$
\lambda\left(\left|u_{h}^{i}\right|^{2 p}\left(u_{h}-u_{h}^{i+1}\right), v_{h}\right) \leq \lambda S_{2} S_{4} S_{8 p}^{2 p}\|f\|_{-1, \Omega}^{2 p}\left|u_{h}^{i+1}-u_{h}\right|_{1, \Omega}\left|v_{h}\right|_{1, \Omega} .
$$

Choosing $v_{h}=u_{h}^{i+1}-u_{h}$ and collecting (4.7) and (4.8) prove the desired estimate. 
4.2. Newton algorithm. Starting from an initial guess $u_{h}^{0}$, construct the sequence $\left(u_{h}^{i}\right)$ in $X_{h}$ such that, for $i \geq 0$, we have

$$
u_{h}^{i+1}=u_{h}^{i}-\left[D F_{h}\left(u_{h}\right)\right]^{-1} \cdot F\left(u_{h}^{i}\right) .
$$

Applying (4.9) to problem (3.3), we obtain the following problem :

Find $u_{h}^{i+1} \in X_{h}$ such that

$$
\forall w_{h} \in X_{h} \quad\left(\nabla u_{h}^{i+1}, \nabla w_{h}\right)+\lambda(2 p+1)\left(\left(u_{h}^{i}\right)^{2 p} u_{h}^{i+1}, w_{h}\right)=2 \lambda p\left(\left(u_{h}^{i}\right)^{2 p+1}, w_{h}\right)+\left\langle f, w_{h}\right\rangle .
$$

The existence and uniqueness of a solution to (4.10) is established due to the Lax-Milgram theorem.

In order to prove the convergence of the Newton's algorithm we apply [7], Chapter 4, Theorem 6.3, which gives

Theorem 4.2. (The convergence Theorem). There exist $\alpha>0$ such that for $h \leq h_{0}$ and an initial guess $u_{h}^{0}$, in the ball with centre $u_{h}$ and radius $\alpha$ the Newton's algorithm (4.10) determines a unique sequence $\left(u_{h}^{i}\right)$ in this ball that converges to the solution $u_{h}$ of problem (3.3). Furthermore the convergence is quadratic:

$$
\left\|u_{h}^{i+1}-u_{h}\right\|_{X} \leq C\left\|u_{h}^{i}-u_{h}\right\|_{X}^{2} .
$$

\section{A POSTERIORI ERROR ANALYSis}

We start this section by introducing some additional notation which is needed for constructing and analyzing the error indicators in the sequel.

For any triangle $K \in \mathcal{T}_{h}$ we denote by $\mathcal{E}(K)$ and $\mathcal{N}(K)$ the set of its edges and vertices respectively and we set

$$
\mathcal{E}_{h}=\bigcup_{K \in \mathcal{T}_{h}} \mathcal{E}(K) \quad \text { and } \quad \mathcal{N}_{h}=\bigcup_{K \in \mathcal{T}_{h}} \mathcal{N}(K)
$$

With any edge $E \in \mathcal{E}_{h}$ we associate a unit vector $n$ such that $n$ is orthogonal to $E$. We split $\mathcal{E}_{h}$ and $\mathcal{N}_{h}$ in the form

$$
\mathcal{E}_{h}=\mathcal{E}_{h, \Omega} \cup \mathcal{E}_{h, \partial \Omega} \quad \text { and } \quad \mathcal{N}_{h}=\mathcal{N}_{h, \Omega} \cup \mathcal{E}_{h, \partial \Omega}
$$

where $\mathcal{E}_{h, \partial \Omega}$ is the set of edges in $\mathcal{E}_{h}$ that lie on $\partial \Omega$ and $\mathcal{E}_{h, \Omega}=\mathcal{E}_{h} \backslash \mathcal{E}_{h, \partial \Omega}$. The same goes for $\mathcal{N}_{h, \partial \Omega}$.

Furthermore, for $K \in \mathcal{T}_{h}$ and $E \in \mathcal{E}_{h}$, let $h_{K}$ and $h_{E}$ be their diameter and length respectively. An important tool in the construction of an upper bound for the total error is Clément's interpolation operator $\mathcal{R}_{h}$ with values in $X_{h}$. The operator $\mathcal{R}_{h}$ satisfies, for all $v \in H_{0}^{1}(\Omega)$, the following local aproximation properties (see R. Verfürth, [11], Chapter 1) :

$$
\begin{aligned}
& \left\|v-R_{h} v\right\|_{L^{2}(K)} \leq C h_{K}|v|_{1, \Delta_{K}}, \\
& \left\|v-R_{h} v\right\|_{L^{2}(E)} \leq C h_{E}^{1 / 2}|v|_{1, \Delta_{E}},
\end{aligned}
$$

where $\Delta_{K}$ and $\Delta_{E}$ are the following sets :

$$
\Delta_{K}=\bigcup\left\{K^{\prime} \in \mathcal{T}_{h} ; \quad K^{\prime} \cap K \neq \emptyset\right\} \quad \text { and } \quad \Delta_{E}=\bigcup\left\{K^{\prime} \in \mathcal{T}_{h} ; K^{\prime} \cap E \neq \emptyset\right\} .
$$

We now recall the following properties (see R. Verfürth, [11], Chapter 1) :

Proposition 5.1. Let $r$ be a positive integer. For all $v \in P_{r}(K)$, the following properties hold

$$
\begin{gathered}
C\|v\|_{L^{2}(K)} \leq\left\|v \psi_{K}^{1 / 2}\right\|_{L^{2}(K)} \leq\|v\|_{L^{2}(K)}, \\
|v|_{1, K} \leq C h_{K}^{-1}\|v\|_{L^{2}(K)} .
\end{gathered}
$$

where $\psi_{K}$ is the triangle-bubble function (equal to the product of the barycentric coordinates associated with the nodes of $K$ ). 
Finally, we denote by $\left[v_{h}\right]$ the jump of $v_{h}$ across the common edge $E$ of two adjacent elements $K, K^{\prime} \in \mathcal{T}_{h}$. We have now provided all prerequisites to establish an upper bound for the total error. Let $u_{h}^{i+1}$ and $u$ be the solution of the iterative problem and the continuous problem respectively. They satisfy the identity

$$
\int_{\Omega} \nabla\left(u_{h}^{i+1}-u\right) \nabla v d \mathbf{x}=\int_{\Omega} \nabla u_{h}^{i+1} \nabla v d \mathbf{x}+\lambda \int_{\Omega}|u|^{2 p} u v d \mathbf{x}-\int_{\Omega} f v d \mathbf{x} .
$$

We now start the a posteriori analysis of the fixed-point algorithm.

5.1. Fixed-point algorithm. In order to prove an upper bound of the error, we first introduce an approximation $f_{h}$ of the data $f$ which is constant on each element $K$ of $\mathcal{T}_{h}$. Then, we distinguish the discretization and linearization errors. We first write the residual equation

$$
\begin{aligned}
& \int_{\Omega} \nabla u \nabla v d \mathbf{x}+\lambda \int_{\Omega}|u|^{2 p} u v d \mathbf{x}-\int_{\Omega} \nabla u_{h}^{i+1} \nabla v d \mathbf{x}-\lambda \int_{\Omega}\left|u_{h}^{i}\right|^{2 p} u_{h}^{i+1} v d \mathbf{x} \\
&=\int_{K}\left(f-f_{h}\right)\left(v-v_{h}\right) d \mathbf{x}+ \sum_{K \in \mathcal{T}_{h}}\left\{\int_{K}\left(f_{h}+\Delta u_{h}^{i+1}-\lambda\left|u_{h}^{i}\right|^{2 p} u_{h}^{i+1}\right)\left(v-v_{h}\right) d \mathbf{x}\right. \\
&\left.-\frac{1}{2} \sum_{E \in \mathcal{E}_{h, \Omega}} \int_{E}\left[\frac{\partial u_{h}^{i+1}}{\partial n}\right]\left(v-v_{h}\right) d \tau\right\},
\end{aligned}
$$

where $\tau$ denotes the tangential coordinate on $\partial K$.

We let the $\Delta u_{h}^{i+1}$ here and afterwards for a better understanding and also in view of the extension to higher order finite elements but it vanishes since we are working with piecewise affine functions.

By adding and subtracting $\lambda \int_{\Omega}\left|u_{h}^{i+1}\right|^{2 p} u_{h}^{i+1} v d \mathbf{x}$, we obtain

$$
\begin{aligned}
\int_{\Omega} \nabla u \nabla v d \mathbf{x}+\lambda \int_{\Omega}|u|^{2 p} u v d \mathbf{x}-\int_{\Omega} \nabla u_{h}^{i+1} \nabla v d \mathbf{x}-\lambda \int_{\Omega}\left|u_{h}^{i+1}\right|^{2 p} u_{h}^{i+1} v d \mathbf{x} \\
=\int_{K}\left(f-f_{h}\right)\left(v-v_{h}\right) d \mathbf{x}+\sum_{K \in \mathcal{T}_{h}}\left\{\int_{K}\left(f_{h}+\Delta u_{h}^{i+1}-\lambda\left|u_{h}^{i}\right|^{2 p} u_{h}^{i+1}\right)\left(v-v_{h}\right) d \mathbf{x}\right. \\
\left.\quad-\frac{1}{2} \sum_{E \in \mathcal{E}_{h, \Omega}} \int_{E}\left[\frac{\partial u_{h}^{i+1}}{\partial n}\right]\left(v-v_{h}\right) d \tau\right\}+\lambda \int_{\Omega}\left(\left|u_{h}^{i}\right|^{2 p}-\left|u_{h}^{i+1}\right|^{2 p}\right) u_{h}^{i+1} v d \mathbf{x} .
\end{aligned}
$$

We now define the local linearization indicator $\eta_{K, i}^{(L)}$ and the local discretization indicator $\eta_{K, i}^{(D)}$ by :

$$
\begin{aligned}
\eta_{K, i}^{(L)} & =\left|u_{h}^{i+1}-u_{h}^{i}\right|_{1, K}, \\
\left(\eta_{K, i}^{(D)}\right)^{2} & =h_{K}^{2}\left\|f_{h}+\Delta u_{h}^{i+1}-\lambda\left|u_{h}^{i}\right|^{2 p} u_{h}^{i+1}\right\|_{L^{2}(K)}^{2}+\sum_{E \in \mathcal{E}_{h, \Omega}} h_{E}\left\|\left[\frac{\partial u_{h}^{i+1}}{\partial n}\right]\right\|_{L^{2}(E)}^{2} .
\end{aligned}
$$

Assumption 5.2. The solution $u_{h}^{i+1}$ of problem (4.1) is such that the operator $I d+S D G\left(u_{h}^{i+1}\right)$ is an isomorphism of $H_{0}^{1}(\Omega)$

Remark 5.3. Owing to the convergence Theorem 4.1, where $C_{1}>0$ and $C_{2} C_{1}^{-1}<1$, Assumption 5.2 is easily derived from the fact that $I d+S D G(u)$ is an isomorphism when $h$ is small enough.

We can now state the first result of this section :

Theorem 5.4. Upper bound. Let $u_{h}^{i+1}$ and $u_{h}$ be the solution of the iterative problem (4.1) and the discrete problem (3.1) respectively. Suppose that the solution $u_{h}^{i+1}$ satisfies Assumption 5.2. Then, there exists a neighbourhood $\mathcal{O}$ of $u$ such that the solution $u_{h}^{i+1}$ in $\mathcal{O}$ satisfies the following a posteriori error estimate

$$
\left|u_{h}^{i+1}-u\right|_{1, \Omega} \leq C\left(\sum_{K \in \mathcal{T}_{h}}\left(\left(\eta_{K, i}^{(D)}\right)^{2}+h_{K}^{2}\left\|f-f_{h}\right\|_{L^{2}(K)}^{2}\right)\right)^{1 / 2}+\left(\sum_{K \in \mathcal{T}_{h}}\left(\eta_{K, i}^{(L)}\right)^{2}\right)^{1 / 2} .
$$


Proof. Owing to Lemma 3.3 and Assumption 5.2, it follows from [10] that, for any $u_{h}^{i+1}$ in a appropriate neighbourhood $\mathcal{O}$ of $u$

$$
\left|u_{h}^{i+1}-u\right|_{1, \Omega} \leq C\left\|u_{h}^{i+1}+\mathcal{S} G\left(u_{h}^{i+1}\right)\right\|_{-1, \Omega} .
$$

By introducing $F(u)$ in (5.6), and from equation (5.5), we obtain

$$
\begin{aligned}
&\left|u_{h}^{i+1}-u\right|_{1, \Omega} \leq C\left(\sup _{v \in H_{0}^{1}(\Omega)} \inf _{v_{h} \in H_{0, h}^{1}(\Omega)} \frac{\left\langle f-f_{h}, v-v_{h}\right\rangle+\left\langle\mathcal{J}, v-v_{h}\right\rangle}{|v|_{1, \Omega}}\right. \\
&\left.+\sup _{v \in H_{0}^{1}(\Omega)} \frac{\lambda \int_{\Omega}\left(\left|u_{h}^{i}\right|^{2 p}-\left|u_{h}^{i+1}\right|^{2 p}\right) u_{h}^{i+1} v d \mathbf{x}}{|v|_{1, \Omega}}\right)
\end{aligned}
$$

where $\left\langle\mathcal{J}, v-v_{h}\right\rangle=\sum_{K \in \mathcal{T}_{h}}\left\{\int_{K}\left(f_{h}+\Delta u_{h}^{i+1}-\lambda\left|u_{h}^{i}\right|^{2 p} u_{h}^{i+1}\right)\left(v-v_{h}\right) d \mathbf{x}\right.$

$$
\left.-\frac{1}{2} \sum_{E \in \mathcal{E}_{h, \Omega}} \int_{E}\left[\frac{\partial u_{h}^{i+1}}{\partial n}\right]\left(v-v_{h}\right) d \tau\right\} .
$$

Using lemma 2.4, we majorate the second term of the right hand side of (5.7) by $\eta_{L}$. Hence, taking $v_{h}$ equal to the Clément operator $\mathcal{R}_{h} v$ in (5.7), we obtain the desired estimate.

We address now the efficiency of the previous indicators.

Theorem 5.5. Lower bound. For each $K \in \mathcal{T}_{h}$, there holds

$$
\begin{aligned}
& \eta_{K, i}^{(L)} \leq\left\|u_{h}^{i}-u\right\|_{1, \omega_{K}}+\left\|u_{h}^{i+1}-u\right\|_{1, \omega_{K}}, \\
& \eta_{K, i}^{(D)} \leq C\left(\left\|u_{h}^{i}-u\right\|_{1, \omega_{K}}+\left\|u_{h}^{i+1}-u\right\|_{1, \omega_{K}}+\sum_{\kappa \subset \omega_{K}} h_{\kappa}\left\|f-f_{h}\right\|_{L^{2}(\kappa)}\right),
\end{aligned}
$$

where $\omega_{K}$ is the union of the triangles sharing at least one edge with $K$.

Proof. The estimation of the linearization indicator follows easily from the triangle inequality by introducing $u$ in $\eta_{K, i}^{(L)}$. We now estimate the discretization indicator $\eta_{K, i}^{(D)}$. We proceed in two steps :

(i) We start by adding and subtracting $\lambda \int_{\Omega}\left|u_{h}^{i}\right|^{2 p} u_{h}^{i+1} v d \mathbf{x}$ and $\lambda \int_{\Omega}\left|u_{h}^{i+1}\right|^{2 p} u_{h}^{i+1} v d \mathbf{x}$ in (5.3). Using the integration by parts we get

$$
\begin{aligned}
& \sum_{K \in \mathcal{T}_{h}} \int_{K}\left(f_{h}+\Delta u_{h}^{i+1}-\lambda\left|u_{h}^{i}\right|^{2 p} u_{h}^{i+1}\right) v d \mathbf{x}=\int_{\Omega} \nabla\left(u_{h}^{i+1}-u\right) \nabla v d \mathbf{x}-\sum_{K \in \mathcal{T}_{h}} \int_{K}\left(f-f_{h}\right) v d \mathbf{x} \\
+ & \frac{1}{2} \sum_{E \in \mathcal{E}_{h, \Omega}} h_{E}^{1 / 2} \int_{E}\left[\frac{\partial u_{h}^{i+1}}{\partial n}\right] v d \tau+\lambda \int_{\Omega}\left(|u|^{2 p} u-\left|u_{h}^{i+1}\right|^{2 p} u_{h}^{i+1}\right) v d \mathbf{x}+\lambda \int_{\Omega} u_{h}^{i+1}\left(\left|u_{h}^{i+1}\right|^{2 p}-\left|u_{h}^{i}\right|^{2 p}\right) v d \mathbf{x} .
\end{aligned}
$$

We choose $v=v_{K}$ such that

$$
v_{K}=\left\{\begin{array}{cl}
\left(f_{h}+\Delta u_{h}^{i+1}-\lambda\left|u_{h}^{i}\right|^{2 p} u_{h}^{i+1}\right) \psi_{K} & \text { sur } K \\
0 & \text { sur } \Omega \backslash K
\end{array}\right.
$$

where $\psi_{K}$ is the triangle-bubble function.

Using Cauchy-Schwarz inequality, (2.1), (5.1) and (5.2) we obtain

$$
\begin{aligned}
& \| f_{h}+\Delta u_{h}^{i+1}- \lambda\left|u_{h}^{i}\right|^{2 p} u_{h}^{i+1} \|_{L^{2}(K)}^{2} \\
& \leq\left(1+\lambda C\|f\|_{-1, \Omega}^{2 p}\right)\left\|u-u_{h}^{i+1}\right\|_{1, K}\left|v_{K}\right|_{1, K}+\left\|f-f_{h}\right\|_{L^{2}(K)}\left\|v_{K}\right\|_{L^{2}(K)} \\
&+\lambda C\|f\|_{-1, \Omega}^{2 p}\left\|u_{h}^{i}-u_{h}^{i+1}\right\|_{1, K}\left|v_{K}\right|_{1, K} .
\end{aligned}
$$

Therefore, we get the following estimate of the first term of the local discretization estimator $\eta_{K, i}^{(D)}$

$$
h_{K}\left\|f_{h}+\Delta u_{h}^{i+1}-\lambda\left|u_{h}^{i}\right|^{2 p} u_{h}^{i+1}\right\|_{L^{2}(K)} \leq C\left(\left\|u-u_{h}^{i+1}\right\|_{1, K}+h_{K}\left\|f-f_{h}\right\|_{L^{2}(K)}\right)+C^{\prime} \eta_{K, i}^{(L)}
$$


where $C^{\prime}=\lambda C\|f\|_{-1, \Omega}^{2 p}$.

(ii) Now we estimate the second term of $\eta_{D, K}$. Similarly, using (5.8) we infer

$$
\begin{array}{r}
\frac{1}{2} \sum_{E \in \mathcal{E}_{h, \Omega}} h_{E}^{1 / 2} \int_{E}\left[\frac{\partial u_{h}^{i+1}}{\partial n}\right] v d \tau=\int_{\Omega} \nabla\left(u-u_{h}^{i+1}\right) \nabla v d \mathbf{x}+\sum_{K \in \mathcal{T}_{h}} \int_{K}\left(f_{h}+\Delta u_{h}^{i+1}-\lambda\left|u_{h}^{i}\right|^{2 p} u_{h}^{i+1}\right) v d \mathbf{x} \\
-\int_{\Omega}\left(f-f_{h}\right) v d \mathbf{x}-\lambda \int_{\Omega}\left(\left|u_{h}^{i+1}\right|^{2 p} u_{h}^{i+1}-\left|u_{h}^{i}\right|^{2 p} u_{h}^{i+1}\right) v d \mathbf{x}-\lambda \int_{\Omega}\left(|u|^{2 p} u-\left|u_{h}^{i+1}\right|^{2 p} u_{h}^{i+1}\right) v d \mathbf{x} .
\end{array}
$$

We choose $v=v_{E}$ such that

$$
v_{E}=\left\{\begin{array}{cl}
L_{E, \kappa}\left(\left[\frac{\partial u_{h}^{i+1}}{\partial n}\right] \psi_{E}\right) & \kappa \in\left\{K, K^{\prime}\right\} \\
0 & \operatorname{sur} \Omega \backslash\left(K \cup K^{\prime}\right)
\end{array}\right.
$$

where $\psi_{E}$ is the edge-bubble function, $K^{\prime}$ denotes the other element of $\mathcal{T}_{h}$ that share $E$ with $K$ and $L_{E, \kappa}$ is a lifting operator from $E$ into $\kappa$ constructed by affine transformation from a fixed lifting operator on the reference element.

Using Cauchy-Schwarz inequality, (2.1), (5.1) and (5.2) we get

$h_{E}^{1 / 2}\left\|\left[\frac{\partial u_{h}^{i+1}}{\partial n}\right]\right\|_{L^{2}(E)}^{2}$

$$
\begin{array}{r}
\leq\left(1+\lambda C\|f\|_{-1, \Omega}^{2 p}\right)\left\|u-u_{h}^{i+1}\right\|_{1, K \cup K^{\prime}}\left\|v_{E}\right\|_{L^{2}(E)}+h_{E}\left\|f-f_{h}\right\|_{L^{2}\left(K \cup K^{\prime}\right)}\left\|v_{E}\right\|_{L^{2}(E)} \\
+h_{E}\left\|f_{h}+\Delta u_{h}^{i+1}-\lambda\left|u_{h}^{i}\right|^{2 p} u_{h}^{i+1}\right\|_{L^{2}\left(K \cup K^{\prime}\right)}\left\|v_{E}\right\|_{L^{2}\left(K \cup K^{\prime}\right)}+C^{\prime} \eta_{K, i}^{(L)}\left|v_{E}\right|_{1, K \cup K^{\prime}} .
\end{array}
$$

Collecting the two bounds above leads to the following estimation

$$
\eta_{K, i}^{(D)} \leq C\left(\left\|u-u_{h}^{i+1}\right\|_{1, \omega_{K}}+\sum_{\kappa \subset \omega_{K}} h_{\kappa}\left\|f-f_{h}\right\|_{L^{2}(\kappa)}\right)+2 C^{\prime} \eta_{K, i}^{(L)} .
$$

Since

we obtain the final result

$$
\eta_{K, i}^{(L)}=\left|u_{h}^{i+1}-u_{h}^{i}\right|_{1, K} \leq\left|u_{h}^{i+1}-u\right|_{1, K}+\left|u_{h}^{i}-u\right|_{1, K},
$$

$$
\eta_{K, i}^{(D)} \leq C\left(\left\|u-u_{h}^{i}\right\|_{1, \omega_{K}}+\left\|u-u_{h}^{i+1}\right\|_{1, \omega_{K}}+\sum_{\kappa \subset \omega_{K}} h_{\kappa}\left\|f-f_{h}\right\|_{L^{2}(\kappa)}\right) .
$$

These estimates of the local linearization and discretization indicators are fully optimal.

5.2. Newton algorithm. Here, our purpose is to analyze the a posteriori estimation error of the Newton algorithm.

We first write the residual equation

$$
\begin{gathered}
\int_{\Omega} \nabla u \nabla v d \mathbf{x}+\lambda(2 p+1) \int_{\Omega}|u|^{2 p} u v d \mathbf{x}-2 \lambda p \int_{\Omega} u^{2 p+1} v d \mathbf{x} \\
-\int_{\Omega} \nabla u_{h}^{i+1} \nabla v d \mathbf{x}-\lambda(2 p+1) \int_{\Omega}\left|u_{h}^{i}\right|^{2 p} u_{h}^{i+1} v d \mathbf{x}+2 \lambda p \int_{\Omega}\left(u_{h}^{i}\right)^{2 p+1} v d \mathbf{x} \\
=\sum_{K \in \mathcal{T}_{h}} \int_{K}\left(f-f_{h}\right)\left(v-v_{h}\right) d \mathbf{x}+\sum_{K \in \mathcal{T}_{h}}\left\{\int_{K}\left(f_{h}+\Delta u_{h}^{i+1}-\lambda(2 p+1)\left|u_{h}^{i}\right|^{2 p} u_{h}^{i+1}+2 \lambda p\left(u_{h}^{i}\right)^{2 p+1}\right)\left(v-v_{h}\right) d \mathbf{x}\right. \\
\left.-\frac{1}{2} \sum_{E \in \mathcal{E}_{h, \Omega}} \int_{E}\left[\frac{\partial u_{h}^{i+1}}{\partial n}\right]\left(v-v_{h}\right) d \tau\right\} .
\end{gathered}
$$

Adding and subtracting $\lambda(2 p+1) \int_{\Omega}\left|u_{h}^{i+1}\right|^{2 p} u_{h}^{i+1} v d \mathbf{x}$ and $2 \lambda p \int_{\Omega}\left(u_{h}^{i+1}\right)^{2 p+1} v d \mathbf{x}$, we obtain 


$$
\begin{gathered}
\int_{\Omega} \nabla u \nabla v d \mathbf{x}+\lambda(2 p+1) \int_{\Omega}|u|^{2 p} u v d \mathbf{x}-2 \lambda p \int_{\Omega} u^{2 p+1} v d \mathbf{x} \\
-\int_{\Omega} \nabla u_{h}^{i+1} \nabla v d \mathbf{x}-\lambda(2 p+1) \int_{\Omega}\left|u_{h}^{i+1}\right|^{2 p} u_{h}^{i+1} v d \mathbf{x}+2 \lambda p \int_{\Omega}\left(u_{h} i+1\right)^{2 p+1} v d \mathbf{x} \\
=\sum_{K \in \mathcal{T}_{h}} \int_{K}\left(f-f_{h}\right)\left(v-v_{h}\right) d \mathbf{x}+\sum_{K \in \mathcal{T}_{h}}\left\{\int_{K}\left(f_{h}+\Delta u_{h}^{i+1}-\lambda(2 p+1)\left|u_{h}^{i}\right|^{2 p} u_{h}^{i+1}+2 \lambda p\left(u_{h}^{i}\right)^{2 p+1}\right)\left(v-v_{h}\right) d \mathbf{x}\right. \\
\left.-\frac{1}{2} \sum_{E \in \Upsilon_{K}} h_{E}^{1 / 2} \int_{E}\left[\frac{\partial u_{h}^{i+1}}{\partial n}\right]\left(v-v_{h}\right) d \tau\right\}+2 \lambda p \int_{\Omega}\left(\left(u_{h}^{i}\right)^{2 p+1}-\left(u_{h}^{i+1}\right)^{2 p+1}\right) v d \mathbf{x} \\
+\lambda(2 p+1) \int_{\Omega} u_{h}^{i+1}\left(\left|u_{h}^{i+1}\right|^{2 p}-\left|u_{h}^{i}\right|^{2 p}\right) v d \mathbf{x} .
\end{gathered}
$$

We define now the linearization estimator $\eta_{L, K}$ and the local discretization indicator $\eta_{K, i}^{(D)}$

$$
\begin{aligned}
\eta_{K, i}^{(L)} & =\left|u_{h}^{i+1}-u_{h}^{i}\right|_{1, K}, \\
\left(\eta_{K, i}^{(D)}\right)^{2} & =h_{K}^{2}\left\|f_{h}+\Delta u_{h}^{i+1}-\lambda(2 p+1)\left|u_{h}^{i}\right|^{2 p} u_{h}^{i+1}+2 \lambda p\left(u_{h}^{i}\right)^{2 p+1}\right\|_{L^{2}(K)}^{2}+\sum_{E \in \mathcal{E}_{h, \Omega}} h_{E}\left\|\left[\frac{\partial u_{h}^{i+1}}{\partial n}\right]\right\|_{L^{2}(E)}^{2}
\end{aligned}
$$

Remark 5.6. Despite their complex aspect, all these indicators are easy to compute.

We consider now the following $C^{1}$ mapping

$$
\begin{aligned}
\widetilde{G}: V & \rightarrow W \\
w & \mapsto \widetilde{G}(w)=f-\lambda(2 p+1)|w|^{2 p} w+2 \lambda p w^{2 p+1},
\end{aligned}
$$

and we set

$$
\widetilde{F}(u)=u-S \widetilde{G}(u)
$$

We consider the finite dimensional approximation of a solution $u \in V$ of the equation $\widetilde{F}(u)=0$.

Lemma 5.7. There exists a neighborhood $\mathcal{V}$ and a real number $\widetilde{L}>0$ such that the following Lipschitz property holds

$$
\forall w \in \mathcal{V}, \quad\|\mathcal{S}(D \widetilde{G}(u)-D \widetilde{G}(w))\|_{\mathcal{L}\left(H_{0}^{1}(\Omega)\right)} \leq \widetilde{L}|u-w|_{1, \Omega}
$$

Proof. We have

$$
\|\mathcal{S}(D \widetilde{G}(u)-D \widetilde{G}(w))\|_{\mathcal{L}\left(H_{0}^{1}(\Omega)\right)} \leq\|D \widetilde{G}(u)-D \widetilde{G}(w)\|_{\mathcal{L}\left(H^{-1}(\Omega)\right)}
$$

We observe that for any $z \in H_{0}^{1}(\Omega)$,

$$
D \widetilde{G}(u) . z-D \widetilde{G}(w) . z=2 \lambda p(2 p+1)\left(|w|^{2 p-1}-|u|^{2 p-1}\right) z-2 \lambda p(2 p+1)\left(|w|^{2 p}-|u|^{2 p}\right) z .
$$

Combining (5.16), (5.17) with Lemma 2.4 yields the desired property.

Assumption 5.8. The solution $u_{h}^{i+1}$ of problem (4.10) is such that the operator $I d+S D \widetilde{G}\left(u_{h}^{i+1}\right)$ is an isomorphism of $H_{0}^{1}(\Omega)$ ( see Remark 5.3).

We can now state the first result of this section :

Theorem 5.9. Upper bound. Let $u$ and $u_{h}^{i+1}$ be the solution of the continuous problem and the iterative problem respectively. We have the following estimation

$$
\left|u_{h}^{i+1}-u\right|_{1, \Omega} \leq\left(\sum_{K \in \mathcal{T}_{h}}\left(\left(\eta_{K, i}^{(D)}\right)^{2}+h_{K}^{2}\left\|f-f_{h}\right\|_{L^{2}(K)}^{2}\right)\right)^{1 / 2}+\left(\sum_{K \in \mathcal{T}_{h}}\left(\eta_{K, i}^{(L)}\right)^{2}\right)^{1 / 2}
$$

where $C$ is a constant that depends on $\lambda, p, f$ and $u_{0}$.

Proof. Owing to Lemma 5.7 and Assumption 5.8, it follows from [10] that, for any $u_{h}^{i+1}$ in a appropriate neighbourhood $\mathcal{O}$ of $u$

$$
\left|u_{h}^{i+1}-u\right|_{1, \Omega} \leq C\left\|u_{h}^{i+1}+\mathcal{S} \widetilde{G}\left(u_{h}^{i+1}\right)\right\|_{-1, \Omega}
$$


By introducing $\widetilde{F}(u)$ in (5.18), and from equation (5.14), we obtain $\left|u_{h}^{i+1}-u\right|_{1, \Omega}$

$\leq C\left(\sup _{v \in H_{0}^{1}(\Omega)} \inf _{v_{h} \in H_{0, h}^{1}(\Omega)} \frac{\left\langle f-f_{h}, v-v_{h}\right\rangle+\left\langle\widetilde{\mathcal{J}}, v-v_{h}\right\rangle}{|v|_{1, \Omega}}+\sup _{v \in H_{0}^{1}(\Omega)} \frac{\lambda(2 p+1) \int_{\Omega}\left(\left|u_{h}^{i}\right|^{2 p}-\left|u_{h}^{i+1}\right|^{2 p}\right) u_{h}^{i+1} v d \mathbf{x}}{|v|_{1, \Omega}}\right.$

$$
\left.+\sup _{v \in H_{0}^{1}(\Omega)} \frac{2 \lambda p \int_{\Omega}\left(\left|u_{h}^{i}\right|^{2 p+1}-\left|u_{h}^{i+1}\right|^{2 p+1}\right) v d \mathbf{x}}{|v|_{1, \Omega}}\right)
$$

where $\left\langle\tilde{\mathcal{J}}, v-v_{h}\right\rangle=\sum_{K \in \mathcal{T}_{h}}\left\{\int_{K}\left(f_{h}+\Delta u_{h}^{i+1}-\lambda(2 p+1)\left|u_{h}^{i}\right|^{2 p} u_{h}^{i+1}+2 \lambda p\left(u_{h}^{i}\right)^{2 p+1}\right)\left(v-v_{h}\right) d \mathbf{x}\right.$

$$
\left.-\frac{1}{2} \sum_{E \in \mathcal{E}_{h, \Omega}} \int_{E}\left[\frac{\partial u_{h}^{i+1}}{\partial n}\right]\left(v-v_{h}\right) d \tau\right\}
$$

We now estimate the last two terms of the right-hand side of equation (5.19).

As previously, $\lambda(2 p+1) \int_{\Omega} u_{h}^{i+1}\left(\left|u_{h}^{i+1}\right|^{2 p}-\left|u_{h}^{i}\right|^{2 p}\right) v d \mathbf{x}$ is estimated as follows

$$
\lambda(2 p+1) \int_{\Omega} u_{h}^{i+1}\left(\left|u_{h}^{i+1}\right|^{2 p}-\left|u_{h}^{i}\right|^{2 p}\right) v d \mathbf{x} \leq \lambda(2 p+1) S^{\prime} C\left|u_{h}^{i+1}-u_{h}^{i}\right|_{1, \Omega}|v|_{1, \Omega}
$$

with $S^{\prime}=4 p S_{2} S_{4} S_{8} S_{8(2 p-1)}^{2 p-1}$.

As well, using Cauchy-Schwarz inequality, Lemma 2.4 and inequality (2.1) yield

$$
2 \lambda p \int_{\Omega}\left(\left(u_{h}^{i}\right)^{2 p+1}-\left(u_{h}^{i+1}\right)^{2 p+1}\right) v d \mathbf{x} \leq 2(2 p+1) C \lambda p S_{2} S_{4}^{2}\left|u_{h}^{i+1}-u_{h}^{i}\right|_{1, \Omega}|v|_{1, \Omega} .
$$

Hence, taking $v_{h}$ equal to the Clément operator $\mathcal{R}_{h} v$, we obtain the desired estimate.

Our purpose now is to prove the efficiency of our indicators.

Theorem 5.10. Lower bound. For each $K \in \mathcal{T}_{h}$, there holds

$$
\begin{aligned}
\eta_{K, i}^{(L)} & \leq\left\|u_{h}^{i}-u\right\|_{1, \omega_{K}}+\left\|u_{h}^{i+1}-u\right\|_{1, \omega_{K}}, \\
\eta_{K, i}^{(D)} & \leq C\left(\left\|u_{h}^{i}-u\right\|_{1, \omega_{K}}+\left\|u_{h}^{i+1}-u\right\|_{1, \omega_{K}}+\sum_{\kappa \subset \omega_{K}} h_{\kappa}\left\|f-f_{h}\right\|_{L^{2}(\kappa)}\right),
\end{aligned}
$$

where $\omega_{K}$ is the union of the triangles sharing at least one edge with $K$.

Proof. The estimation of the linearization indicator follows easily by introducing $u$ in $\eta_{K, i}^{(L)}$. We now start by estimating the discretization indicator $\eta_{K, i}^{(D)}$. We proceed in two steps :

(i) Adding and subtracting $2 \lambda p \int_{\Omega}\left|u_{h}^{i}\right|^{2 p+1} v d \mathbf{x}, \lambda(2 p+1) \int_{\Omega}\left|u_{h}^{i}\right|^{2 p} u_{h}^{i+1} v d \mathbf{x}$ and $\lambda(2 p+1) \int_{\Omega}\left|u_{h}^{i}+1\right|^{2 p} u_{h}^{i+1} v d \mathbf{x}$ in (5.3) and using integration by parts leads to

$$
\begin{gathered}
\sum_{K \in \mathcal{T}_{h}} \int_{K}\left(f_{h}+\Delta u_{h}^{i+1}+\lambda(2 p+1)\left|u_{h}^{i}\right|^{2 p} u_{h}^{i+1}-2 \lambda p\left(u_{h}^{i}\right)^{2 p+1}\right) v d \mathbf{x}=\int_{\Omega} \nabla\left(u_{h}^{i+1}-u\right) \nabla v d \mathbf{x}-\sum_{K \in \mathcal{T}_{h}} \int_{K}\left(f-f_{h}\right) v d \mathbf{x} \\
-\frac{1}{2} \sum_{E \in \mathcal{E}_{h, \Omega}} h_{E}^{1 / 2} \int_{E}\left[\frac{\partial u_{h}^{i+1}}{\partial n}\right] v d \tau+\lambda \int_{\Omega}\left(|u|^{2 p} u-\left|u_{h}^{i+1}\right|^{2 p} u_{h}^{i+1}\right) v d \mathbf{x}+\lambda(2 p+1) \int_{\Omega} u_{h}^{i+1}\left(\left|u_{h}^{i+1}\right|^{2 p}-\left|u_{h}^{i}\right|^{2 p}\right) v d \mathbf{x} \\
+2 \lambda p \int_{\Omega}\left(\left(u_{h}^{i}\right)^{2 p+1}-\left(u_{h}^{i+1}\right)^{2 p+1}\right) v d \mathbf{x} .
\end{gathered}
$$


We choose $v=v_{K}$ in (5.22) where

$$
v_{K}=\left\{\begin{array}{cl}
\left(f_{h}+\Delta u_{h}^{i+1}+\lambda(2 p+1)\left|u_{h}^{i}\right|^{2 p} u_{h}^{i+1}-2 \lambda p\left(u_{h}^{i}\right)^{2 p+1}\right) \psi_{K} & \text { sur } K \\
0 & \text { sur } \Omega \backslash K
\end{array}\right.
$$

and $\psi_{K}$ is the triangle-bubble function.

Using Cauchy-Schwarz inequality, (2.1), (5.1) and (5.2) we obtain

$$
\begin{gathered}
\left\|\left(f_{h}+\Delta u_{h}^{i+1}+\lambda(2 p+1)\left|u_{h}^{i}\right|^{2 p} u_{h}^{i+1}-2 \lambda p\left(u_{h}^{i}\right)^{2 p+1}\right)\right\|_{L^{2}(K)}^{2} \\
\leq(1+\lambda C)\left\|u-u_{h}^{i+1}\right\|_{1, K}\left|v_{K}\right|_{1, K}+\left\|f-f_{h}\right\|_{L^{2}(K)}\left\|v_{K}\right\|_{L^{2}(K)} \\
+\lambda C(4 p+1)\left\|u_{h}^{i}-u_{h}^{i+1}\right\|_{1, K}\left|v_{K}\right|_{1, K} .
\end{gathered}
$$

Hence, we get the following estimation of the first term of the local discretization estimator $\eta_{K, i}^{(D)}$

$h_{K}\left\|\left(f_{h}+\Delta u_{h}^{i+1}+\lambda(2 p+1)\left|u_{h}^{i}\right|^{2 p} u_{h}^{i+1}-2 \lambda p\left(u_{h}^{i}\right)^{2 p+1}\right)\right\|_{L^{2}(K)}$

$$
\leq C\left(\left\|u-u_{h}^{i+1}\right\|_{1, K}+h_{K}\left\|f-f_{h}\right\|_{L^{2}(K)}\right)+\lambda C(4 p+1) \eta_{K, i}^{(L)} .
$$

(ii) We now estimate the second term of $\eta_{K, i}^{(D)}$. Similarly, using (5.22) we infer

$$
\begin{aligned}
& \frac{1}{2} \sum_{E \in \mathcal{E}_{h, \Omega}} h_{E}^{1 / 2} \int_{E}\left[\frac{\partial u_{h}^{i+1}}{\partial n}\right] v d \tau \\
& =\int_{\Omega} \nabla\left(u-u_{h}^{i+1}\right) \nabla v d \mathbf{x}+\sum_{K \in \mathcal{T}_{h}} \int_{K}\left(f_{h}+\Delta u_{h}^{i+1}+\lambda(2 p+1)\left|u_{h}^{i}\right|^{2 p} u_{h}^{i+1}-2 \lambda p\left(u_{h}^{i}\right)^{2 p+1}\right) v d \mathbf{x} \\
& \quad-\int_{\Omega}\left(f-f_{h}\right) v d \mathbf{x}-\lambda \int_{\Omega}\left(|u|^{2 p} u-\left|u_{h}^{i+1}\right|^{2 p} u_{h}^{i+1}\right) v d \mathbf{x}+\lambda(2 p+1) \int_{\Omega} u_{h}^{i+1}\left(\left|u_{h}^{i+1}\right|^{2 p}-\left|u_{h}^{i}\right|^{2 p}\right) v d \mathbf{x} \\
& \quad+2 \lambda p \int_{\Omega}\left(\left(u_{h}^{i}\right)^{2 p+1}-\left(u_{h}^{i+1}\right)^{2 p+1}\right) v d \mathbf{x} .
\end{aligned}
$$

We choose $v=v_{E}$

$$
v_{E}=\left\{\begin{array}{cl}
L_{E, \kappa}\left(\left[\frac{\partial u_{h}^{i+1}}{\partial n}\right] \psi_{E}\right) & \kappa \in\left\{K, K^{\prime}\right\} \\
0 & \operatorname{sur} \Omega \backslash\left(K \cup K^{\prime}\right)
\end{array}\right.
$$

with the same notation as in the proof of Theorem 5.5.

Using Cauchy-Schwarz inequality, (2.1), (5.1) and (5.2) we get

$$
\begin{gathered}
h_{E}^{1 / 2}\left\|\left[\frac{\partial u_{h}^{i+1}}{\partial n}\right]\right\|_{L^{2}(E)}^{2} \leq(1+\lambda C)\left\|u-u_{h}^{i+1}\right\|_{1, K \cup K^{\prime}}\left\|v_{E}\right\|_{L^{2}(E)}+h_{E}\left\|f-f_{h}\right\|_{L^{2}\left(K \cup K^{\prime}\right)}\left\|v_{E}\right\|_{L^{2}(E)} \\
+h_{E}\left\|\left(f_{h}+\Delta u_{h}^{i+1}+\lambda(2 p+1)\left|u_{h}^{i}\right|^{2 p} u_{h}^{i+1}-2 \lambda p\left(u_{h}^{i}\right)^{2 p+1}\right)\right\|_{L^{2}\left(K \cup K^{\prime}\right)}\left\|v_{E}\right\|_{L^{2}\left(K \cup K^{\prime}\right)} \\
+\lambda C(4 p+1) \eta_{K, i}^{(L)}\left|v_{E}\right|_{1, K \cup K^{\prime}} .
\end{gathered}
$$

Collecting the two above bounds leads to the following estimation

$$
\eta_{K, i}^{(D)} \leq C\left(\left\|u-u_{h}^{i+1}\right\|_{1, \omega_{K}}+\sum_{\kappa \subset \omega_{K}} h_{\kappa}\left\|f-f_{h}\right\|_{L^{2}(\kappa)}\right)+2 \lambda C(4 p+1) \eta_{K, i}^{(L)} .
$$

Since

we obtain the final result

$$
\eta_{K, i}^{(L)}=\left|u_{h}^{i+1}-u_{h}^{i}\right|_{1, K} \leq\left|u_{h}^{i+1}-u\right|_{1, K}+\left|u_{h}^{i}-u\right|_{1, K},
$$

$$
\eta_{K, i}^{(D)} \leq C\left(\left\|u-u_{h}^{i}\right\|_{1, \omega_{K}}+\left\|u-u_{h}^{i+1}\right\|_{1, \omega_{K}}+\sum_{\kappa \subset \omega_{K}} h_{\kappa}\left\|f-f_{h}\right\|_{L^{2}(\kappa)}\right) .
$$




\section{Numerical RESUlts}

In this section, we present numerical experiments for our nonlinear problem. These simulations have been performed using the code FreeFem ++ due to F. Hecht and O. Pironneau, see [8]. They are divided into five main categories :

(1) a priori and a posteriori estimates for the fixed-point algorithm

(2) a priori and a posteriori estimates for the Newton algorithm

(3) comparison of the two algorithms

(4) mixed algorithm

(5) conclusion.

\subsection{Fixed point algorithm.}

6.1.1. A priori estimation. A first test case. We consider the domain $\Omega=] 0,1\left[^{2}\right.$, each edge is divided into $N$ equal segments so that $\Omega$ is divided into $N^{2}$ equal squares. We consider the theoretical solution $u=x(x-1) y(y-1)\left(x^{2}+y^{2}\right) \mathrm{e}^{x y}$ and the iterative algorithm 4.1.

We use the classical stopping criterion $\operatorname{err}_{L} \leq 10^{-7}$, where $\operatorname{err}_{L}$ is defined by

$$
\operatorname{err}_{L}=\frac{\left|u_{h}^{i+1}-u_{h}^{i}\right|_{1, \Omega}}{\left|u_{h}^{i+1}\right|_{1, \Omega}}
$$

The convergence of our nonlinear problem depends on two parameters $\lambda$ and $p$. In fact, big values of $|u|^{2 p}$ and $\lambda$ lead to a divergence of our problem. Indeed, Theorem 4.1 ensures the convergence of the solution of the iterative problem for small values of $\lambda$ and $p$.

Figure 1 compares the exact solution with the estimated solution for $N=120, \lambda=1$ and $p=1$.
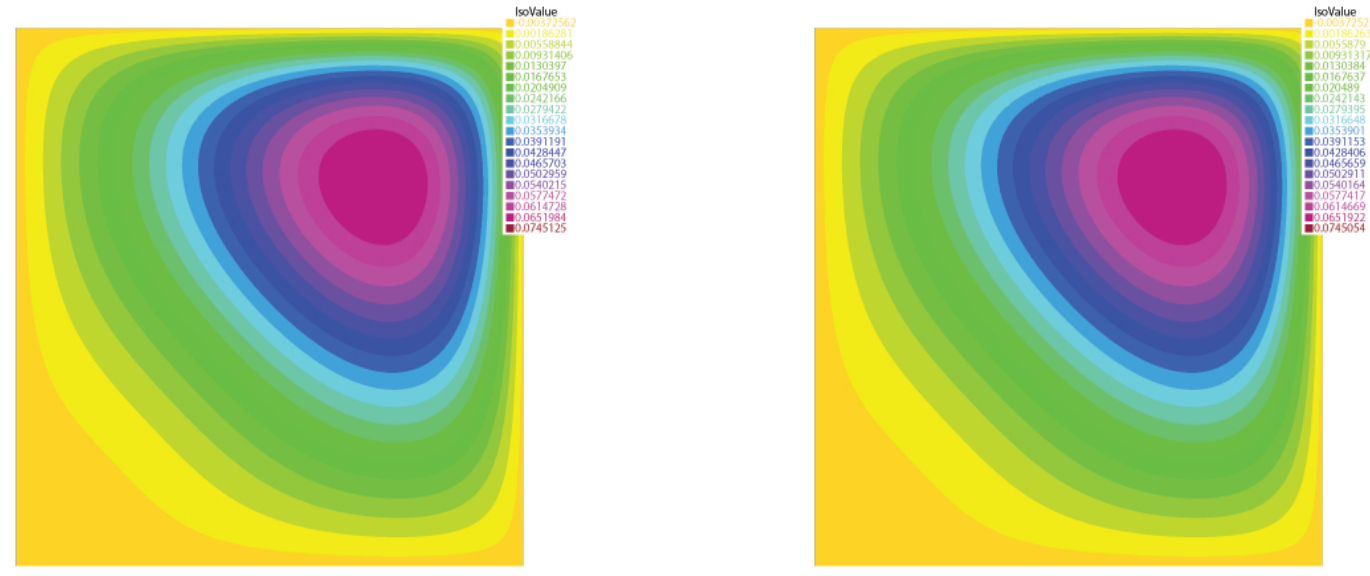

Figure 1. Exact solution (left) and numerical solution (right) for $N=120, \lambda=$ 1 and $p=1$.

Figure 2 presents the error curve as a function of $h$ in logarithmic scales. We test the algorithm for the number of segments $N$ going from 80 to 120 with $\lambda=1$ and $p=1$. The slope is equal to 1.018. 


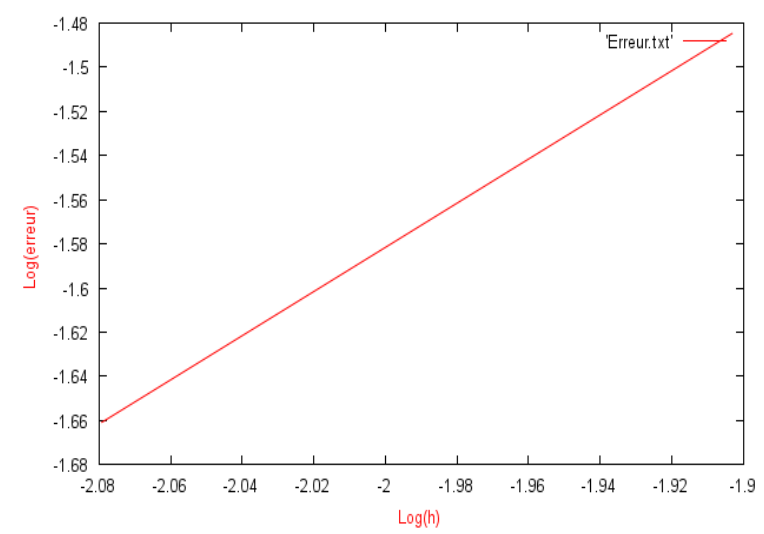

Figure 2. Error curve for $N$ going from 80 to 120 with $\lambda=1$ and $p=1$.

6.1.2. A posteriori analysis. A second test case. In this section, we test our a posteriori error estimates on our model problem. We consider the domain $\Omega=]-1,1\left[2\right.$, the exact solution $u=\mathrm{e}^{-100\left(x^{2}+y^{2}\right)}$ and the same iterative algorithm as in the first test case. We define two different stopping criteria : the classical one defined previously and the new criterion $\operatorname{err}_{L} \leq \gamma \eta_{i}^{(D)}$, with $\gamma$ a positive parameter and $\eta_{i}^{(D)}$ defined by

$$
\eta_{i}^{(D)}=\left(\sum_{K \in \mathcal{T}_{h}}\left(\eta_{K, i}^{(D)}\right)^{2}\right)^{1 / 2} .
$$

We give in Figure 3 a comparison of the estimated solution and the exact solution on an adaptively refined mesh for $\lambda=2$ and $p=1$.
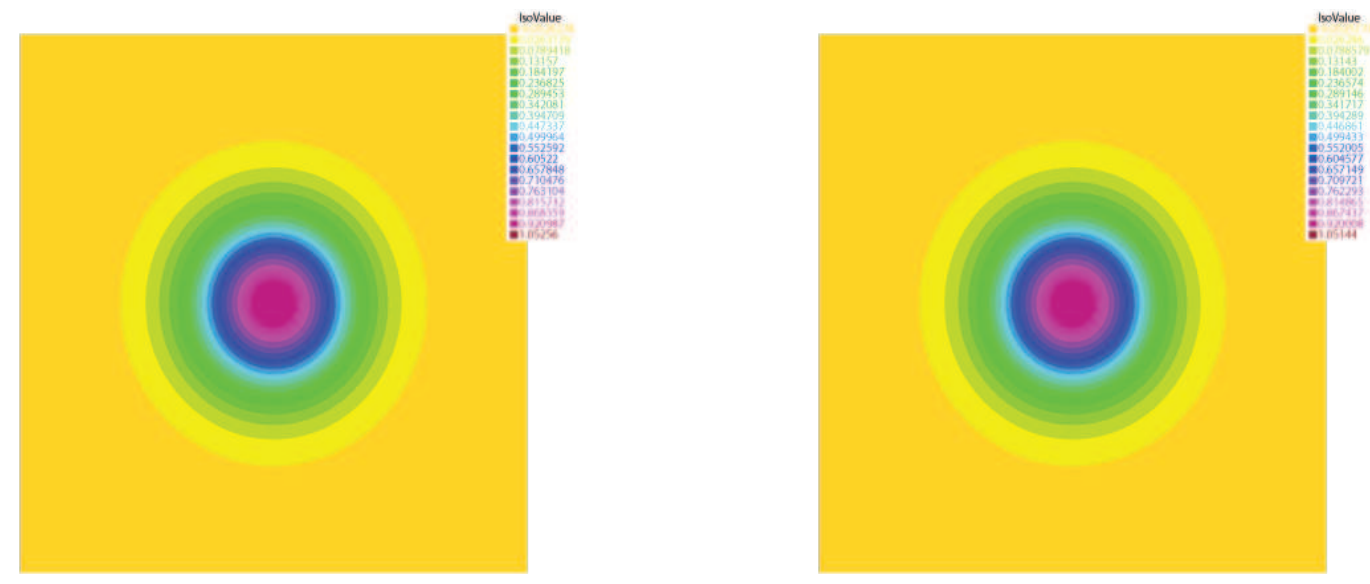

Figure 3. Exact solution (left) and numerical solution (right) for $\lambda=2$ and $p=1$.

Figures 4 to 7 show the evolution of the mesh (see [11], Introduction) using the fixed-point algorithm with the new stopping criterion. In fact, an adaptive mesh refinement can be outlined as follows :

For $i \geq 0$,

(1) Construct an initial mesh $\mathcal{T}_{i}$

(2) Solve the discrete problem on $\mathcal{T}_{i}$

(3) For each element $K$ in $\mathcal{T}_{i}$ compute the a posteriori error estimate. 
(4) If the estimated global error is sufficiently small then STOP. Otherwise refine the mesh and return to step (2).

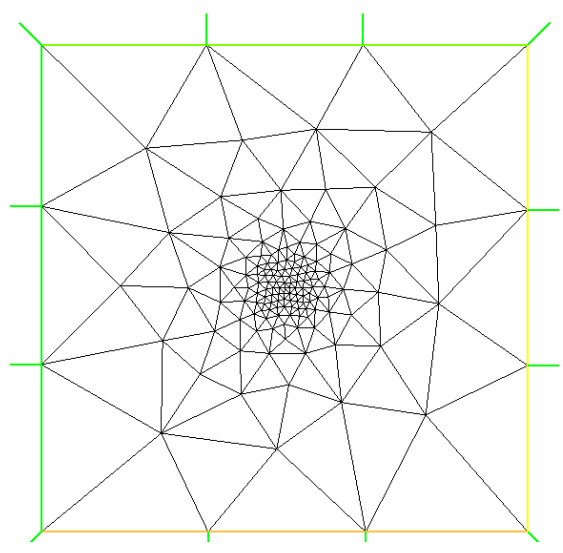

Figure 4. Mesh refinement for $\gamma=0.01: 148$ vertices

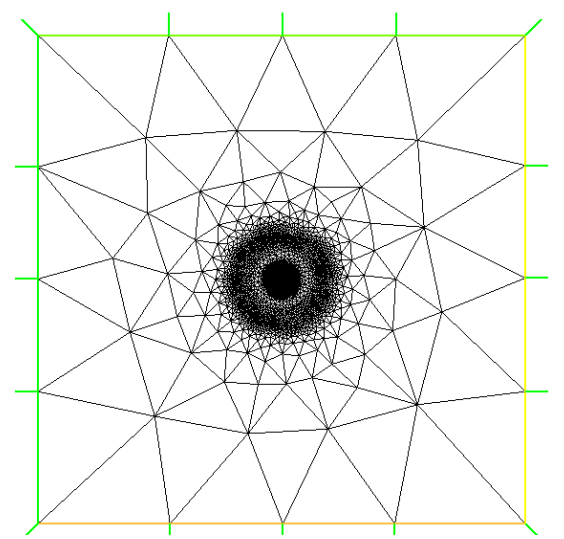

Figure 6 . Mesh refinement for $\gamma=0.01: 5083$ vertices

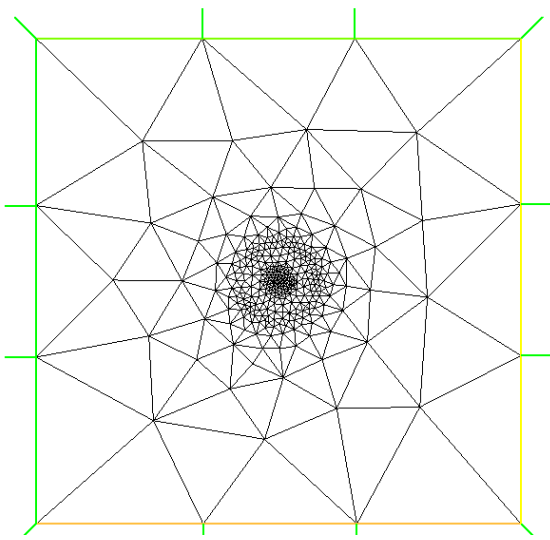

FiguRE 5. Mesh refinement for $\gamma=0.01: 334$ vertices

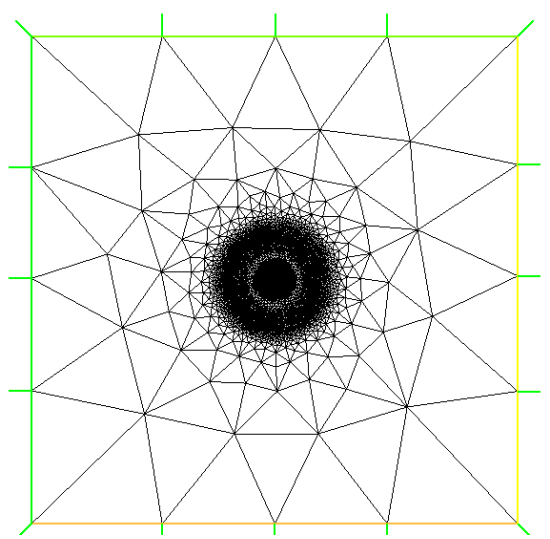

Figure 7. Mesh refinement for $\gamma=0.01: 9963$ vertices

Figure 8 presents the error curve for uniform (red) and adaptive (blue) mesh refinement using the new stopping criterion with $\lambda=50, p=10$ and $\gamma=10^{-3}$. We note that the error using an adaptive mesh is much smaller than the error using an uniform mesh.

Figure 9 illustrates the performance of our new stopping criterion with $\gamma=0.01$ by comparing it to the classical stopping criterion $\operatorname{err}_{L} \leq 10^{-5}$. We can clearly observe that our new stopping criterion reduces the number of iterations. 


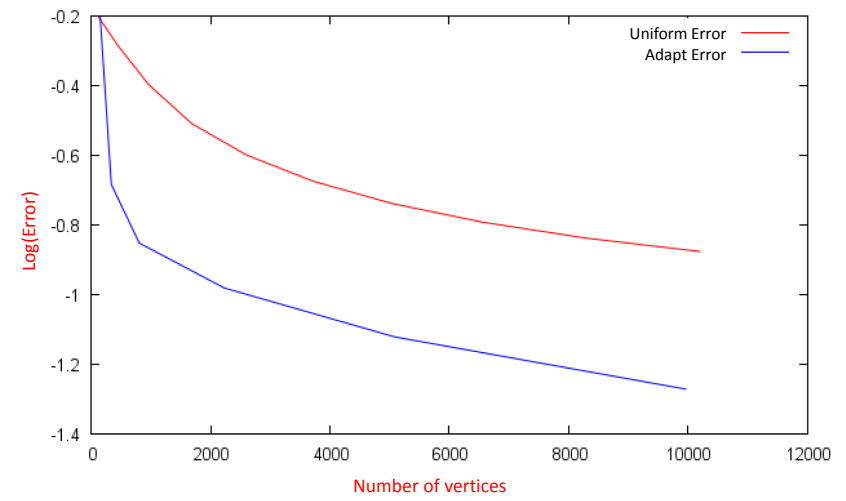

Figure 8. Error curve as a function of the vertices number for $\lambda=50$ and $p=10$. Uniform error (top), adaptive error (bottom).

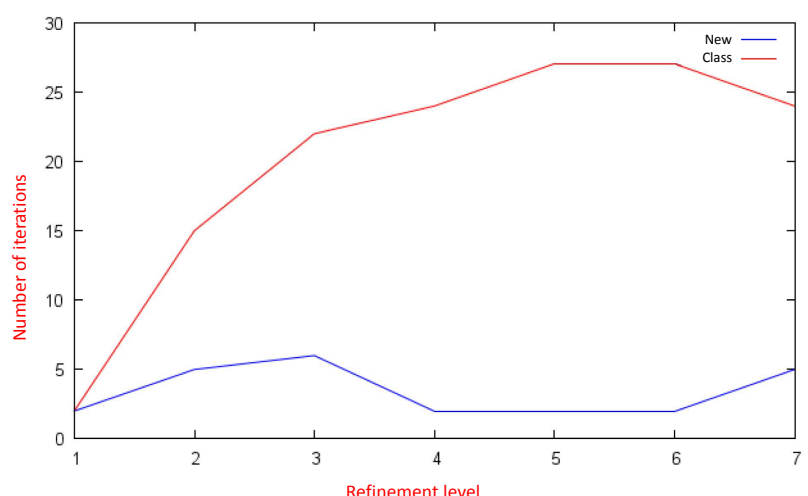

Figure 9. Iterations number as a function of the refinement level for $\lambda=50$ and $p=10$. Classical criterion (top), new criterion (bottom).

\subsection{Newton algorithm.}

6.2.1. A priori analysis. A first test case. In this section we consider the domain $\Omega=] 0,1[2$ with the theoretical solution $u=x(x-1) y(y-1)\left(x^{2}+y^{2}\right) \mathrm{e}^{x y}$.

We consider the iterative algorithm (4.10) and the classical stopping criterion $\operatorname{err}_{L}=\frac{\left|u_{h}^{i+1}-u_{h}^{i}\right|_{1, \Omega}}{\left|u_{h}^{i+1}\right|_{1, \Omega}} \leq$ $10^{-7}$.

Figure 10 presents the error curve as a function of $h$ in logarithmic scales. We test the algorithm for $N$ going from 80 to 120 with $\lambda=1$ and $p=1$. The slope is equal to 0.999 .

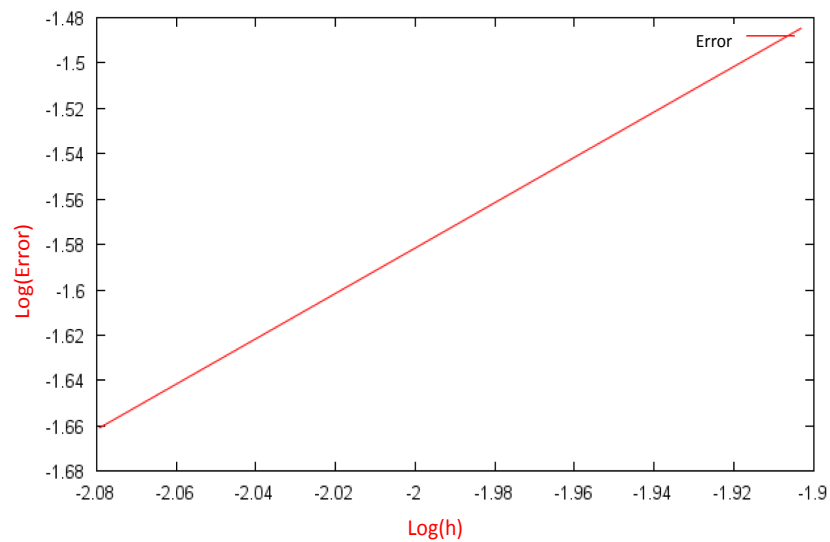

Figure 10. Error curve for $N$ going from 80 to 120 with $\lambda=1$ and $p=1$.

In Table 1, we represent the error estimates that correspond to the mesh $N=200$. 
Remark 6.1. In the sequel, we denote by div the divergence of the scheme (this means that the error of the algorithm does not decrease).

\begin{tabular}{|l||c|c|c|c|c|c|}
\hline$\lambda$ & 1 & 2 & 3 & 4 & 5 & 10 \\
\hline \hline $10^{-2}$ & 0.0131062 & 0.0131053 & 0.0131053 & 0.0131053 & 0.0131053 & 0.0131053 \\
\hline $10^{-1}$ & 0.0131145 & 0.0131053 & 0.0131053 & 0.0131053 & 0.0131053 & 0.0131053 \\
\hline 1 & 0.0131053 & 0.0131056 & 0.0131053 & 0.0131053 & 0.0131053 & 0.0131053 \\
\hline 5 & 0.0131058 & 0.0131069 & 0.0131053 & 0.0131053 & 0.0131053 & 0.0131053 \\
\hline 10 & 0.0131073 & 0.0131085 & 0.0131053 & 0.0131053 & 0.0131053 & 0.0131053 \\
\hline 50 & 0.0131058 & 0.0131053 & 0.0131053 & 0.0131053 & 0.0131053 & 0.0131053 \\
\hline $10^{2}$ & 0.0131095 & 0.0131053 & 0.0131053 & 0.0131053 & 0.0131053 & 0.0131053 \\
\hline $10^{3}$ & div & 0.0131053 & 0.0131053 & 0.0131053 & 0.0131053 & 0.0131053 \\
\hline $10^{4}$ & div & 0.0131055 & 0.0131053 & 0.0131053 & 0.0131053 & 0.0131053 \\
\hline $10^{5}$ & div & div & 0.013127 & 0.0131053 & 0.0131053 & 0.0131053 \\
\hline $10^{6}$ & div & div & 0.0153185 & 0.0131053 & 0.0131053 & 0.0131053 \\
\hline $10^{7}$ & div & div & div & 0.0131053 & 0.0131053 & 0.0131053 \\
\hline $10^{8}$ & div & div & div & 0.0131053 & 0.0131053 & 0.0131053 \\
\hline $10^{9}$ & div & div & div & div & 0.0131053 & 0.0131053 \\
\hline $10^{10}$ & div & div & div & div & 0.0131053 & 0.0131053 \\
\hline
\end{tabular}

TABle 1. Error estimates for $N=200$.

6.2.2. A posteriori analysis. A second test case. In this section, we test our a posteriori error estimates on our model problem. We consider the domain $\Omega=]-1,1\left[^{2}\right.$, the exact solution $u=\mathrm{e}^{-100\left(x^{2}+y^{2}\right)}$ and the same iterative algorithm as in the first test case. We define two different stopping criteria :

$$
\begin{aligned}
\eta_{L} & \leq \eta^{*}, \\
\eta_{L} & \leq \gamma \eta_{i}^{(D)},
\end{aligned}
$$

with $\gamma$ a positive parameter and with the same definition for $\eta_{i}^{(D)}$ as previously.

Figure 11 compares the exact solution with the estimated solution for $\lambda=10$ and $p=1$.
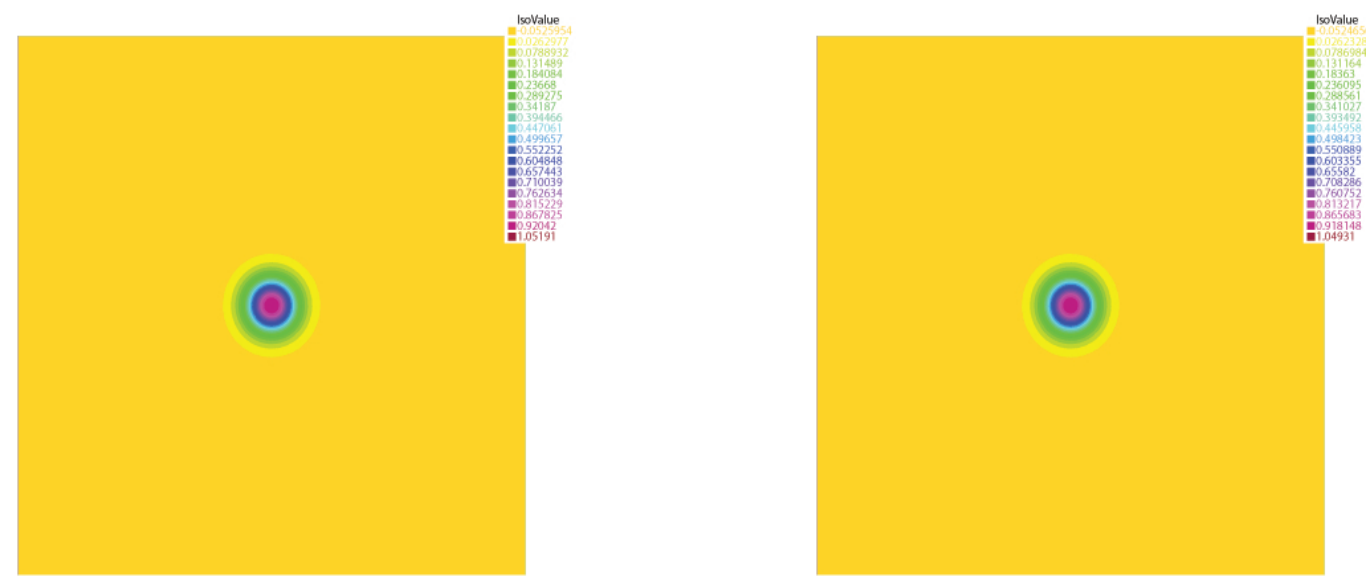

Figure 11. Exact solution (left) and numerical solution (right) after the mesh refinement for $\lambda=10$ and $p=1$. 
Figures 12 to 15 show the evolution of the mesh using the Newton algorithm with the new stopping criterion $\left(\eta_{L} \leq \gamma \eta_{i}^{(D)}\right)$.

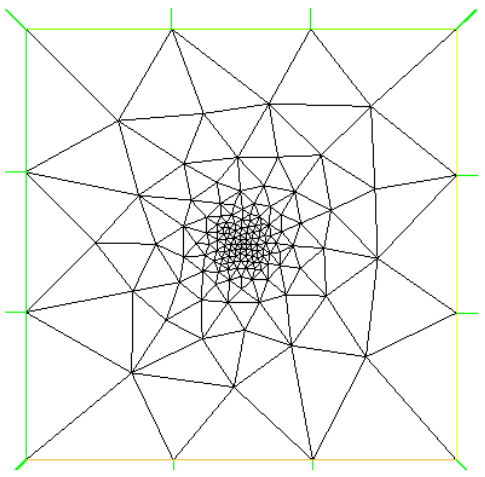

FiguRE 12. Mesh refinement : 147 vertices

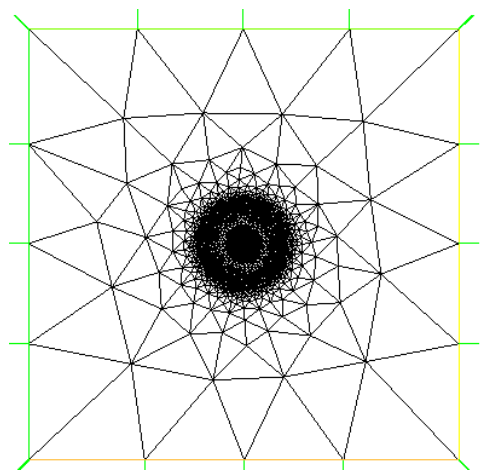

FiguRE 14. Mesh refinement : 4896 vertices

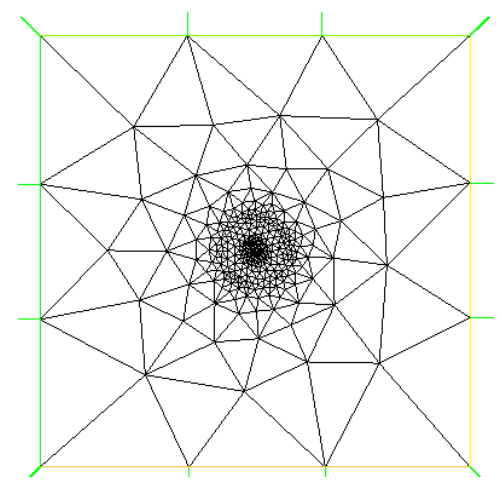

Figure 13. Mesh refinement : 320 vertices

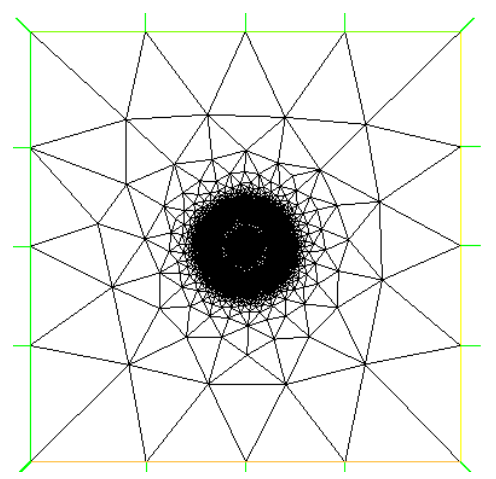

FigURE 15. Mesh refinement : 9599 vertices

Figure 16 presents the error curve for uniform (red) and adaptive (blue) mesh refinement using the new stopping criterion with $\lambda=10, p=1$ and $\gamma=10^{-3}$. We note that the error using an adaptive mesh is much smaller than the error using an uniform mesh.

Finally, Figure 17 illustrate the performance of our new stopping criterion for the Newton iteration by comparing it to a more classical stopping criterion, 


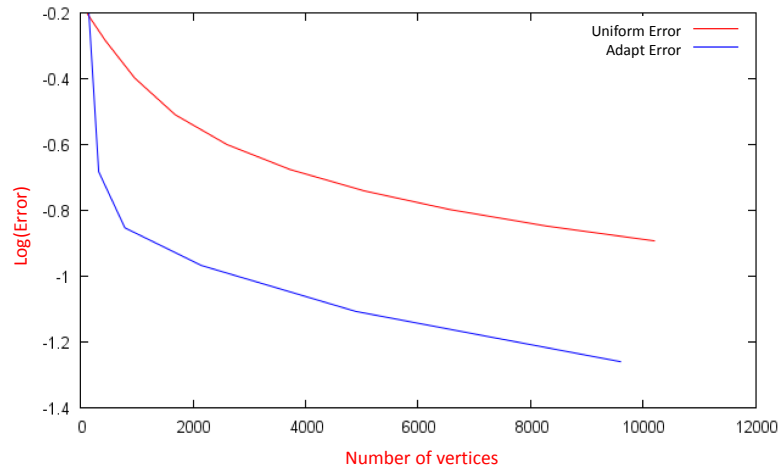

Figure 16. Error curve as a function of the vertices number for $\lambda=10$ and $p=1$. Uniform error (top), adaptive error (bottom).

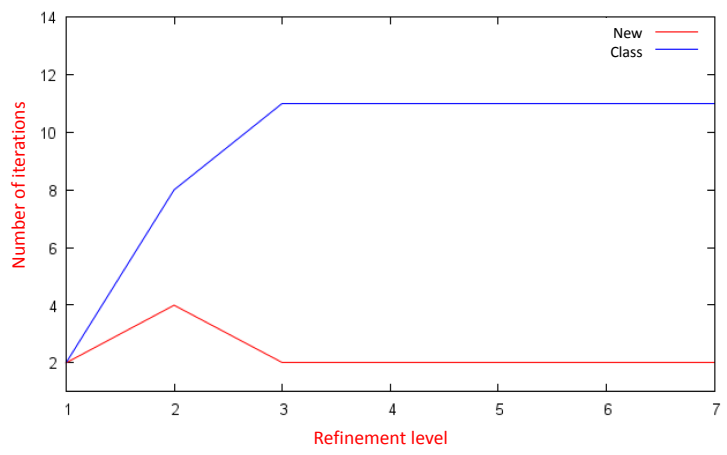

Figure 17. Number of iterations as a function of the refinement level for $\lambda=10$ and $p=1$. Classical criterion (top), new criterion (bottom).

6.3. Comparison of the algorithms. In this section our purpose is to compare the fixed-point algorithm with the Newton algorithm using adaptively refined meshes. Figure 18 illustrate the performance of the Newton algorithm with an initial guess $u_{h}^{0}=0.03$. When comparing the two methods, Newton's method requires only two iterations for all considered refinement level, whereas the fixed-point algorithm requires 10 iterations in the first refinement level and does not go beneath 4 for the rest levels. It is clear that the method of Newton avoids performing an excessive number of iterations.

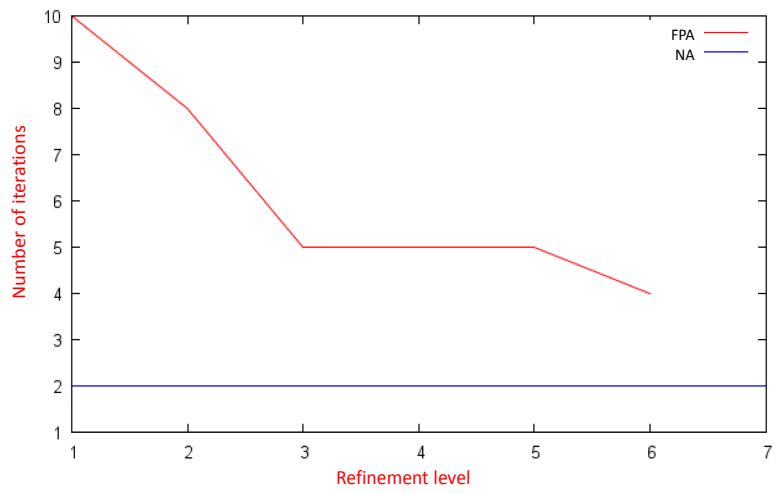

FIgURE 18. Number of iterations for the new stopping criterion on adaptively refined meshes with $\gamma=0.001$. FPA algorithm (top), Newton's algorithm (bottom).

6.4. Mixed Algorithm. As we saw in this section, the main advantage of the Newton's algorithm is that it converges faster than the fixed-point algorithm. However, the choice of the initial data in Newton's method is quite important, an initial guess $u_{h}^{0}$ that is not close to the theoretical solution can lead to the divergence of our problem. Thus, in order to eliminate this drawback we propose a mixed algorithm. In fact, we benefit from the advantages of both algorithms. We start the first iteration by the fixed-point method and we continue the iterative process using the Newton's algorithm. The following table provides the CPU time of the convergence of each algorithm. 


\begin{tabular}{|c|c|c|c|}
\hline Initial data $u_{h}^{0}$ & Newton algorithm & Fixed point algorithm & Mixed algorithm \\
\hline 0.5 & $3.33 \mathrm{~s}$ & $8.145 \mathrm{~s}$ & $4.866 \mathrm{~s}$ \\
1.5 & div & $8.142 \mathrm{~s}$ & $4.812 \mathrm{~s}$ \\
1.6 & div & $8.149 \mathrm{~s}$ & $4.81 \mathrm{~s}$ \\
1.7 & div & $8.162 \mathrm{~s}$ & $4.787 \mathrm{~s}$ \\
1.8 & div & $8.147 \mathrm{~s}$ & $4.796 \mathrm{~s}$ \\
1.9 & div & $8.163 \mathrm{~s}$ & $4.797 \mathrm{~s}$ \\
2 & div & $8.149 \mathrm{~s}$ & $4.799 \mathrm{~s}$ \\
2.5 & div & $8.145 \mathrm{~s}$ & $4.784 \mathrm{~s}$ \\
\hline
\end{tabular}

TABLE 2. CPU time in seconds of the convergence of each algorithm.

6.5. Conclusion. We have presented in this work an applied treatment of a posteriori error estimation for finite element approximation of the nonlinear problem (1.1). In order to solve the discrete problem we proposed two different methods : the fixed-point algorithm and the Newton algorithm. Thus, two sources of error appear, the linearization error and the discretization error. Balancing these two errors is very important; in fact, it avoid performing an excessive number of iterations. As well, this analysis can be applied for many other nonlinear problems. Furthermore, we have compared the two different iteration algorithms; in fact, the Newton's algorithm converges faster than the fixed-point algorithm. However, the choice of the initial data in Newton's method is quite important. The results are presented in Table 2 .

\section{RÉFÉRENCES}

[1] Adams R.A., Sobolev Spaces, Acadamic Press, INC, 1978.

[2] Babuška I., Rheinboldt W.C., Error estimates for adaptive finite element computations, SIAM J. Numer. Anal. 4 (1978), 736-754.

[3] Brezzi F., Rappaz J., Raviart P.-A., Finite dimensional approximation of nonlinear problems, Part I : Branches of nonsingular solutions, Numer. Math. 36 (1980), 1-25.

[4] Chaillou A.-L., Suri M., Computable error estimators for the approximation of nonlinear problems by linearized models, Computable Methods in Applied Mechanics and Engineering 196 (2006), 210-224.

[5] Chaillou A.-L., Suri M., A posteriori estimation of the linearization error for strongly monotone nonlinear operators, Computable Methods in Applied Mechanics and Engineering 205 (2007), 72-87.

[6] El Alaoui L., Ern A.,Vohralík M., Guaranteed and robust a posteriori error estimate and balancing discretization and linearization errore for monotone non linear problems, Computable Methods in Applied Mechanics and Engineering 200 (2011), 2782-2795.

[7] Girault V., Raviart P.-A., Finite Element Methods for Navier-Stokes Equations, Springer-Verlag, 1986.

[8] Hecht F., New development in FreeFem++, Journal of Numerical Mathematics 20 (2012), 251-266.

[9] Kavian O., Introduction à la théorie des points critiques et applications aux problèmes elliptiques, Springer-Verlag, 1993.

[10] Pousin J., Rappaz J., Consistency, stability, a priori and a posteriori errors for Petrov-Galerkin methods applied to nonlinear problems, Numer. Math. 69 (1994), no. 2, 213-231.

[11] Verfürth R., A Posteriori Error Estimation Techniques For Finite Element Methods, Numerical Mathematics And Scientific Computation, Oxford, 2013. 